\title{
G-FONCTIONS ET COHOMOLOGIE DES HYPERSURFACES SINGULIËRES
}

\author{
Cristiana Bertolin
}

Our object of study is the arithmetic of the differential modules $\mathcal{W}^{(l)}(l \in \mathbb{N}-$ $\{0\}$ ), associated by Dwork's theory to a homogeneous polynomial $f(\lambda, X)$ with coefficients in a number field. Our main result is that $\mathcal{W}^{(l)}$ is a differential module of type $G$, c'est-à-dire, a module whose solutions are $G$-functions. For the proof we distinguish two cases: the regular one and the non regular one.

Our method gives us an effective upper bound for the global radius of $\mathcal{W}^{(l)}$, which doesn't depend on " $l$ " but only on the polynomial $f(\lambda, X)$. This upper bound is interesting because it gives an explicit estimate for the coefficients of the solutions of $\mathcal{W}^{(l)}$.

In the regular case we know there is an isomorphism of differential modules between $\mathcal{W}^{(1)}$ and a certain De Rham cohomology group, endowed with the GaussManin connection, c'est-à-dire, our module "comes from geometry". Therefore our main result is a particular case of André's theorem which assert that at least in the regular case, all modules coming from geometry are of type $G$.

\section{INTRODUCTION}

Dans cet article on va étudier l'arithmétique des modules différentiels $\mathcal{W}^{(l)}$ introduits par Dwork dans ses travaux sur la fonction Zêta d'une hypersurface [3, 4, 5]. L'arrière-plan de cette étude est la conjecture de Dwork et Bombieri qui affirme l'existence d'une représentation intégrale algébrique pour toute $G$-fonction (au sens de Siegel). Plus précisément, dans [7, Section 12] Dwork fait correspondre à toute fonction hypergéométrique ${ }_{k} F_{k-1}$ un polynôme à plusieurs variables puis, par une construction algébrique élémentaire, un module différentiel $\mathcal{W}$ (analogue à notre $\mathcal{W}^{(1)}$ ), qui a la propriété d'être isomorphe à la cohomologie associée à la différentielle qui apparaît dans la formule intégrale pour ${ }_{k} F_{k-1}$. Dans [9] on peut trouver des tables où on associe de même un tel polynôme aux éléments de la liste de Horn, aux quatre séries de Lauricella et à leurs variantes confluentes.

Received 23rd April, 1996

Ce travail est déduit de ma thèse de "Laurea" que j'ai rédigée à Padoue sous la direction de Bernard Dwork et de Francesco Baldassarri. Je tiens à les remercier de m'avoir proposé ce sujet et de m'avoir soutenue pendant toute la durée de ce travail. Je tiens également à remercier Yves André et Gilles Christol qui m'ont aidée, avec beaucoup de patience, a continuer et à approfondir cette étude sur les $G$-fonctions.

Copyright Clearance Centre, Inc. Serial-fee code: 0004-9729/97 \$A2.00+0.00. 
Partant d'un polynôme homogène $f(\lambda, X) \in R=\sum K(\lambda) X^{u}$, où $\lambda$ est un élément transcendant sur $K$ corps de nombres, $u \in \mathbb{N}^{n+1}$ et $\sum u_{i} \equiv 0 \bmod d$, avec $d \in \mathbb{N}$ fixé, on définit, comme Dwork, pour chaque $l \in \mathbb{N}-\{0\}$, le $K(\lambda)$-espace

$$
R / \sum_{\left(i_{1}, \ldots, i_{l}\right) \in\{1, \ldots, n+1\}^{l}} D_{i_{1}} \cdots D_{i_{l}} R,
$$

où $D_{i}=E_{i}+f_{i}$ avec $E_{i}=X_{i} \frac{\partial}{\partial X_{i}}$ et $f_{i}=E_{i} f$ pour chaque $i \in\{1, \ldots, n+1\}$. Le résultat principal de ce travail est le suivant:

THÉoRÈme. Pour chaque $l \in \mathbb{N}-\{0\}, \mathcal{W}^{(l)}$, muni de la connexion $\sigma_{\lambda}=\frac{\partial}{\partial \lambda}+\frac{\partial f}{\partial \lambda}$, est un module différentiel de type $G$, c'est-à-dire, un module dont toutes les solutions sont des $G$-fonctions.

Pour vérifier ce résultat, on va distinguer deux cas:

CAS $1 . l \geqslant 1$ et $f_{1}, \ldots, f_{n+1}$ est une suite régulière (c'est-à-dire, $\sum_{i=1}^{k} f_{i} h_{i}=0$ avec $1 \leqslant$ $k \leqslant n+1$ et $h_{i} \in K(\lambda)[X]$ pour $1 \leqslant i \leqslant k$, implique que $h_{i}=\sum_{j=1}^{k} \eta_{i, j} f_{j}$ pour chaque $i=1, \ldots, k$, avec $\eta_{i, j} \in K(\lambda)[X]$ et $\eta_{i, j}=-\eta_{j, i}$ pour chaque $\left.i, j=1, \ldots, k\right)$. Le cas $l=1$ fut traité par Dwork dans un cours tenu à Padoue pendant l'année universitaire 1993/94. Puisque ce cours n'a pas été publié, sa démonstration sera reproduite dans la Section 2. Si $l>1, \mathcal{W}^{(l)}$ est une extension itérée de copies de $\mathcal{W}^{(1)}$ et le résultat est une simple conséquence du cas $l=1$.

Les cas "réguliers" les plus connus sont fournis par les fonctions hypergéometriques ${ }_{k} F_{k-1}$. Bien que pour tout $k>2$ les polynômes associés à ces fonctions ne forment pas une suite régulière, on peut trouver dans [7] une construction qui permet de se ramener au cas "régulier": dans [7, Section 12], Dwork construit un anneau $\widehat{R}$ par rapport auquel ces polynômes forment une suite régulière. Après, grâce aux résultats de [7, Section 9.7], on sait que le module $\mathcal{W}^{(1)}$ construit à partir de $R$, et le module $\widehat{\mathcal{W}}^{(1)}$ construit à partir de $\widehat{R}$, sont, sous certaines hypothèses raisonnables, isomorphes.

CAS $2 . l \geqslant 1$ et $f_{1}, \ldots, f_{n+1}$ n'est pas une suite régulière. Dans ce cas la démonstration est beaucoup plus longue et compliquée car elle fait intervenir la "théorie de déformation" qui permet de se ramener au cas "régulier" grâce à un argument de spécialisation (voir 3.2).

Rappelons que l'une des caractérisations des modules différentiels de type $G$ est la finitude du "rayon global $\rho$ " (voir [1, IV Section 3]). Nos méthodes nous fournissent en fait une majoration effective du rayon global de $\mathcal{W}^{(l)}$, qui ne dépend pas de "l " mais 
seulement du polynôme $f(\lambda, X)$ :

$$
\begin{aligned}
& \rho\left(\mathcal{W}^{(l)}\right) \leqslant \\
& d^{2 n}\left(\begin{array}{c}
n+(n+1) d \\
n
\end{array}\right)^{2}\left[\frac{3}{2} \log \left(\left(\begin{array}{l}
n+d \\
d-2
\end{array}\right) \frac{4(n+2 d)}{(d-1)}\right)+\frac{3}{4}\left(\log (m+1)+h_{\infty}(f)\right)\right]
\end{aligned}
$$

où

(1) $n+1$ est le nombre de variables $X_{1}, \ldots, X_{n+1}$ de $f(\lambda, X)$,

(2) $d$ est le degré d'homogénéité en les variables $X_{1}, \ldots, X_{n+1}$ de $f(\lambda, X)$,

(3) $m$ est le degré en $\lambda$ de $f(\lambda, X)$,

(4) $h_{\infty}(f)$ est la hauteur logarithmique à l'infini de $f(\lambda, X)$.

Cette majoration effective est intéressante car, grâce à [1, V Section 5 et Section 6 ], elle nous fournit une majoration explicite pour le commun dénominateur des $m$-premiers coefficients d'une $G$-fonction solution de $\mathcal{W}^{(l)}$ et ce genre d'estimations effectives des coefficients est très utile pour la théorie diophantienne des valeurs des $G$-fonctions.

Grâce au livre [7], les résultats de ce travail se généralisent sans difficulté aux polynômes quasi-homogènes de la forme $g(X)=\sum_{j=1}^{n_{2}} X_{n_{1}+j} f^{(j)}\left(X_{1}, \ldots, X_{n_{1}}\right)$, où $n_{1}, n_{2} \in \mathbb{N}$ et les $f^{(j)}$ sont des formes de degré $d_{j}$ en les variables $X_{1}, \ldots, X_{n_{1}}$, qui sont associés dans ce livre aux fonctions hypergéométriques.

L'interprétation cohomologique de $\mathcal{W}^{(1)}$, sous l'hypothèse de régularité, a été étudiée par Katz: dans [10], il démontre que $\mathcal{W}^{(1)}$ est isomorphe, en tant que module différentiel, au n-ième espace de cohomologie de De Rham de l'hypersurface $Z(f(\lambda, X))$ pour $n$ impair (et à un quotient explicite de cet espace pour $n$ pair), muni de la connexion de Gauss-Manin. Cet isomorphisme de notre module différentiel avec un module de Picard-Fuchs entraine l'origine geométrique des $\mathcal{W}^{(l)}$; par conséquent, sous l'hypothèse de régularité, notre résultat principal est un cas particulier du théorème d'André qui affirme qu'au moins dans le cas régulier, tous les modules différentiels d'origine géométrique sont de type $G$ (voir [1, V Appendice]).

Une autre démonstration de ce théorème peut se déduire du travail de André et Baldassarri sur la stabilité de la notion de $G$-connexion par le foncteur image directe [2]. Il faut toutefois observer que notre démonstration est beaucoup plus explicite car, comme on l'a déjà dit, elle permet de trouver une majoration effective du rayon global de $\mathcal{W}^{(l)}$.

\section{TERminologie Et NOtATIONS}

\subsection{Valeurs absolues.}


$\mathcal{O}_{K}$

$\mathcal{P}$ (respectivement $\mathcal{P}_{f}, \mathcal{P}_{\infty}$ ) $K_{v}$

$\mathbb{Q}_{p}$

$\mathbb{C}_{\text {p }}$

$\mathbb{Z}_{p}$

$\pi$

$t_{v}$

$|\cdot|_{\mathrm{cl}}$

$\|\vec{w}\|=\left(\sum_{1 \leqslant i \leqslant n}\left|w_{i}\right| c c^{2}\right)^{1 / 2}$

$\operatorname{ord}_{v} \alpha=-\frac{\log |\alpha|_{v}}{\log p}$

$h_{\infty}(g)=\sum_{v \in \mathcal{P}_{\infty}} \sup _{i} \log ^{+}\left|a_{i}\right|_{v}$

$|g|_{0}(r)=\sup _{\substack{x \in K \\|x|=r}}|g(x)|_{v}$ anneau des entiers de $K$

ensemble des places (respectivement finies, infinies) de $K$

complété de $K$ pour la valeur absolue $v$-adique complété de $\mathbb{Q}$ pour la valeur absolue $p$-adique complété de la clôture algébrique de $\mathbb{Q}_{p}$ pour la valeur absolue $p$-adique

anneau des entiers $p$-adiques

élément de $\mathbb{C}_{p}$ tel que $\pi^{p-1}=-p$

point générique de $v \in \mathcal{P}_{f}$ dans le sens de Dwork (voir [8, III 5])

valeur absolue euclidienne

$\forall \vec{w}=\left(w_{1}, \ldots, w_{n}\right) \in K^{n}$

$\forall \alpha \in K, v \in \mathcal{P}_{f}, v \mid p$

$\forall g(X)=\sum_{i=0}^{m} a_{i} \lambda^{i} \in K[X]$

hauteur logarithmique infinie de $g(X)$

$\forall v \in \mathcal{P}_{f}$ et $g(X) \in K((X))$ convergente pour $|x|_{v}=r$

\subsection{Normalisation Des VAleurs aBsolues.}

(0.2.1) si $v \in \mathcal{P}_{f}$ et $v \mid p$, alors $|p|_{v}=p^{-l_{v} / l}$ avec $l_{v}=\left[K_{v}: \mathbb{Q}_{v}\right]$;

(0.2.2) si $v \in \mathcal{P}_{\infty}$, alors $\forall \alpha \in K \quad|\alpha|_{v}=|\alpha|_{\text {cl }}^{\varepsilon_{v} / l}$ avec $\varepsilon_{v}=1$ si $K_{v}=\mathbb{R}$ et $\varepsilon_{v}=2$ si $K_{v}=\mathbb{C}$.

\subsection{LeS MODULES $\mathcal{W}^{(l)}$.}

Fixons $d, m \in \mathbb{N}-\{0\}$. Soient $\lambda$ un élément transcendant sur $K, R$ l'espace sur $K(\lambda)$ engendré par $X^{u}=X_{1}^{u_{1}} \cdots X_{n+1}^{u_{n+1}}$, où $u \in \mathbb{N}^{n+1}$ et $u_{1}+\cdots+u_{n+1} \equiv 0$ $\bmod d$, et pour tout $s \in \mathbb{N}, R_{s}$ le sous-espace de $R$ engendré par les monômes $X^{u}$ avec $u \in \mathbb{N}^{n+1}$ et $u_{1}+\cdots+u_{n+1}=s d$. On observe que $R=\bigoplus_{s \in \mathbb{N}} R_{s}$. De plus soit $f(\lambda, X) \in R$ un polynôme homogène de degré $d$ dans les $n+1$ variables $X_{1}, \ldots, X_{n+1}$ et de degré $m$ en $\lambda$ :

$$
f(\lambda, X)=\sum_{u_{1}+\cdots+u_{n+1}=d} A_{u}(\lambda) X^{u}, \quad A_{u}(\lambda) \in K(\lambda) .
$$

Pour simplifier les calculs on suppose que $f(\lambda, X) \in \mathcal{O}_{K}[\lambda, X]$ et pour simplifier les notations on pose

$$
\mathcal{F}=\left\{u \in \mathbb{N}^{n+1} \mid u_{1}+\cdots+u_{n+1}=\mathrm{d}\right\}
$$


Soient pour $i=1, \ldots, n+1, E_{i}=x_{i} \frac{\partial}{\partial x_{i}}, f_{i}=E_{i} f, D_{i}=E_{i}+f_{i}$ et pour tout $v \in \mathbb{N}^{n+1}, D^{v}=D_{1}^{v_{1}} \cdots D_{n+1}^{v_{n+1}}$. Avec cette terminologie on peut finalement définir pour chaque $l \in \mathbb{N}-\{0\}$ les $K(\lambda)$-modules $\mathcal{W}^{(l)}$ :

$$
\mathcal{W}^{(l)}=R / \sum_{|v|=l} D^{v} R \quad \forall l \in \mathbb{N}-\{0\}
$$

Pour conclure on observe que l'application $K$-linéaire $\sigma_{\lambda}=\frac{\partial}{\partial \lambda}+\frac{\partial f}{\partial \lambda}$ commute avec $D_{i}$ pour chaque $i \in\{1, \ldots, n+1\}$, et par conséquent pour chaque $l \in \mathbb{N}-\{0\}$ elle induit une connexion sur $\mathcal{W}^{(l)}$ qui prolonge l'action de $d /(d \lambda)$ sur $K(\lambda)$.

\section{THÉORIE GÉNÉRIQUE}

1.0. Avant de démontrer les deux cas enoncés dans l'introduction, on va vérifier des résultats "génériques" qui nous servirons pour étudier les modules $\mathcal{W}^{(l)}$. J'utilise l'adjectif "générique" car ces résultats concernent le polynôme générique, c'est-à-dire, un polynôme homogène de degré $d, h(A, X)=\sum_{u \in \mathcal{F}} A_{u} X^{u} \in \mathbb{Q}\left(A_{u}\right)_{u \in \mathcal{F}}[X]$, tel que, les $\left\{A_{u}\right\}_{u \in \mathcal{F}}$ soient $\left(\begin{array}{c}n+d \\ n\end{array}\right)$-éléments algébriquement indépendants sur $\mathbb{Q}$. Mais si on spécialise de façon adéquate les coefficients de ce polynôme, on obtient le polynôme $f(\lambda, X)$, qu'on a introduit en 0.3 , et par conséquent les résultats de ce paragraphe se spécialisent en des résultats concernant le polynôme $f(\lambda, X)$, dont on aura besoin dans la suite.

1.1. Pour simplifier les notations, soient $A$ le vecteur $\left(\ldots, A_{u}, \ldots\right)_{u \in \mathcal{F}}, K_{0}$ le corps $\mathbb{Q}(\pi)$ muni de la valeur absolue qui prolonge la valeur absolue $p$-adique de $\mathbb{Q}$ et enfin $\Omega_{0}$ le corps $K_{0}(A)$ muni de la norme de Gauss par rapport à $A$ (voir $[8,14]$ ). De plus pour $i=1, \ldots, n+1$, posons $h_{i}=E_{i} h$. Avec ces notations on peut énoncer une propriété très importante du polynôme générique: $h_{1}, \ldots, h_{n+1}$ est une suite régulière. En effet:

Proposition 1.2. Soit $h(A, X)=\sum_{u \in \mathcal{F}} A_{u} X^{u}$ un polynôme générique homogène de degré $d$. Si $R\left(h, h_{1}, \ldots, h_{n}\right)$ indique le résultant des polynômes $h, h_{1}, \ldots, h_{n}$, alors

$$
\left|R\left(h, h_{1}, \ldots, h_{n}\right)\right|=1 .
$$

Preuve: Pour simplifier les notations nous indiquons avec $\mathrm{R}(\mathrm{A})$ le résultant des polynômes $h, h_{1}, \ldots, h_{n}$. Puisque $R(A) \in \mathcal{O}_{\Omega_{0}}$ il suffit de vérifier que $R(\bar{A}) \neq 0$, où $R(\bar{A})=R\left(\bar{h}, \bar{h}_{1}, \ldots, \bar{h}_{n}\right)$ avec $\bar{h}=\sum_{u \in \mathcal{F}} \bar{A}_{u} X^{u}$ et les $\bar{A}_{u}$ appartenant au corps résiduel de $\Omega_{0}$. 
Commençons par observer que pour chaque $p$ il existe un polynôme homogène $g(X) \in \mathbb{F}_{p}[X]$ tel que $g, \frac{\partial g}{\partial X_{1}}, \ldots, \frac{\partial g}{\partial X_{n+1}}$ n'aient pas de zéros communs. En effet il suffit de prendre

(1) si $p \nmid d$

$$
g(X)=X_{1}^{d}+\cdots+X_{n+1}^{d}
$$

(2.1) si $p \mid d$ et $d>2$

$$
g(X)=X_{1}^{d}+X_{1} X_{2}^{d-1}+X_{2} X_{3}^{d-1}+\cdots+X_{n-1} X_{n}^{d-1}+X_{n} X_{n+1}^{d-1} ;
$$

(2.2.1) si $p \mid d, d=2$ et $n+1$ est pair

$$
g(X)=\left(X_{1}^{2}+X_{1} X_{2}+X_{2}^{2}\right)+\cdots+\left(X_{n}^{2}+X_{n} X_{n+1}+X_{n+1}^{2}\right)
$$

(2.2.2) si $p \mid d, d=2$ et $n+1$ est impair

$$
g(X)=\left(X_{1}^{2}+X_{1} X_{2}+X_{2}^{2}\right)+\cdots+\left(X_{n}^{2}+X_{n} X_{n+1}+X_{n+1}^{2}\right)+X_{n+1}^{2} .
$$

Mais alors si on choisit $\mathrm{n}+1$ hyperplans génériques $\left\{e_{i}(X)=0, i=1, \ldots, n+1\right\}$, on sait que pour tout $r \in\{1, \ldots, n+1\},\left\{g, e_{j_{1}}, \ldots, e_{j_{r}}\right\}_{1 \leqslant j_{i} \leqslant n+1}$ est une variété non singulière. Par conséquent, en faisant un changement de coordonnées, on trouve que pour chaque $r \in\{1, \ldots, n+1\},\left\{g, X_{j_{1}}, \ldots, X_{j_{r}}\right\}_{\substack{1 \leqslant j_{i} \leqslant n+1 \\ 1 \leqslant i \leqslant r}}$ est une variété non singulière. Supposons maintenant par l'absurde que $R(\bar{A})=0$, c'est-à-dire, que

$$
\left\{\bar{h}, X_{1} \frac{\partial \bar{h}}{\partial X_{1}}, \ldots, X_{n} \frac{\partial \bar{h}}{\partial X_{n}}\right\}
$$

aient un zéro commun. Il existe alors une partition de $\{1, \ldots, n+1\},\left\{j_{1}, \ldots, j_{r}\right\}$ $\bigcup\left\{i_{1}, \ldots, i_{n+1-r}\right\}$ avec $0 \leqslant r \leqslant n+1$, telle que la variété $\left\{\bar{h}, X_{j_{1}}, \ldots, X_{j_{r}}\right\}_{1 \leqslant j_{i} \leqslant n+1}$ soit une hypersurface singulière de $\mathbb{P}_{K_{0}}^{n-r}$. Donc si on choisit une spécialisation de $A$, soit $A^{(0)}$, telle que $\bar{h}\left(\bar{A}^{(0)}, X\right)=g(X)$, on obtient que $\left\{g, X_{j_{1}}, \ldots, X_{j_{r}}\right\}$ est une hypersurface singulière de $\mathbb{P}_{K_{0}}^{n-r}$. Mais ceci est en contradiction avec ce qui précède. [ REMARque 1.3. Normalement on voit que $h_{1}, \ldots, h_{n+1}$ forment une suite régulière à ce que le résultant de ces polynômes n'est pas nul. Dans cette proposition on a considéré $R\left(h, h_{1}, \ldots, h_{n}\right)$ à la place de $R\left(h_{1}, \ldots, h_{n+1}\right)$ à cause du problème suivant: d'après le théorème de Euler sur les fonctions homogènes on sait que $d h=\sum_{i=1}^{n+1} h_{i}$. Mais alors pour tous les $p$ qui divisent $d$, les fonctions $h_{i}$ sont linéairement dépendantes modulo $p$ 
et donc le résultant $R\left(h_{1}, \ldots, h_{n+1}\right)$ est identiquement nul modulo $p$. C'est pour éviter ce problème qu'on considère le résultant $R\left(h, h_{1}, \ldots, h_{n}\right)$ en observant que tout zéro commun dans $\mathbb{P}_{K_{0}}^{n}$ de $h, h_{1}, \ldots, h_{n}$, est un zéro commun des polynômes $h_{1}, \ldots, h_{n+1}$ et vice versa.

1.4. Les définitions suivantes sont analogues à celles de la Section 1 , mais ici, le rôle qu'avait $f(\lambda, X)$, sera joué par $h(A, X)$. Soit $R_{A}$ l'espace sur $\Omega_{0}$ engendré par $X^{u}$, où $u \in \mathbb{N}^{n+1}$ et $u_{1}+\cdots+u_{n+1} \equiv 0 \bmod d$, et pour tout $l \in \mathbb{N}-\{0\}$, soit $\mathcal{W}_{A}^{(l)}=R_{A} / \sum_{|v|=l} D_{A}^{v} R_{A}$, où pour chaque $v \in \mathbb{N}^{n+1}, D_{A}^{v}=D_{A, 1}^{v_{1}} \cdots D_{A, n+1}^{v_{n+1}}$ avec

$$
D_{A, i}=E_{i}+h_{i}(A, X) \quad(1 \leqslant i \leqslant n+1) .
$$

Puisque d'après $1.2 h_{1}, \ldots, h_{n+1}$ forment une suite régulière, on a grâce à [ 5 , Lemme -1.2 et Théorème 2.1$]$

$$
\begin{aligned}
& \operatorname{dim}_{\Omega_{0}} \mathcal{W}_{A}^{(1)}=d^{n} \stackrel{d e f f}{=} N \\
& \operatorname{dim}_{\Omega_{0}} \mathcal{W}_{A}^{(l)}=\sum_{i=0}^{l-1}\left(\begin{array}{c}
n+i \\
n
\end{array}\right) N \stackrel{\text { def }}{=} \bar{N} .
\end{aligned}
$$

1.5. Pour rappeler certaines notions concernant l'espace dual de $R_{A}$, on va suivre l'exposition de [5, Section 3], mais on peut trouver une présentation analogue aussi dans [7, 3.2]. Soit $R_{A}^{*}$ l'espace dual de $R_{A}$. Ses éléments peuvent être vus comme éléments de l'anneau $\left\{\sum_{u \in \mathcal{F}} B_{u}\left(1 / X^{u}\right) \mid B_{u} \in \Omega_{0}\right\}$. La dualité est définie de la façon suivante:

$$
\begin{aligned}
\langle., .\rangle: R_{A}^{*} \times R_{A} & \longrightarrow \Omega_{0} \\
\left(\xi^{*}, \xi\right) & \longmapsto \text { coefficient de } X^{0} \text { dans le produit } \xi^{*} \cdot \xi .
\end{aligned}
$$

Pour $i=1, \ldots, n+1$, l'adjointe de l'opérateur différentiel $D_{A, i}$ est

$$
D_{A, i}^{*}=\gamma_{-} \circ\left(-E_{i}+h_{i}(A, X)\right)
$$

où

$$
\gamma_{-} \frac{1}{X^{v}}=\left\{\begin{array}{ll}
\frac{1}{X^{v}} & \text { si } v_{i} \geqslant 0 \\
0 & \text { autrement. }
\end{array} \quad \forall i=1, \ldots, n+1,\right.
$$

De plus pour tout $i \in \mathbb{N}-\{0\}$, soit $\mathcal{K}_{A}^{(i)}=\left\{\xi^{*} \in R_{A}^{*}\left|D_{A}^{* w} \xi^{*}=0 \quad \forall\right| w \mid=i\right\}$ le sous espace de $R_{A}^{*}$ qui annule $\sum_{|w|=i} D_{A}^{w} R_{A}$.

1.6. Dans ce paragraphe on va étudier l'espace $\mathcal{K}_{A}^{(l)}$ pour tout $l \in \mathbb{N}-\{0\}$. Mais avant d'énoncer le résultat principal concernant cet espace, on va changer un peu les 
notations et on va rappeler la définition de certains espaces utilisés par Dwork dans ses travaux sur la fonction Zêta d'une hypersurface. Pour simplifier les calculs on va introduire $\pi_{v} X_{0}$, c'est-à-dire, on va remplacer $X_{i}$ par $X_{i}\left(\pi_{v} X_{0}\right)^{1 \backslash d}$. À cause de ce changement on doit substituer à l'ensemble $\mathcal{F}$, défini en (0.3.1), l'ensemble:

$$
\mathcal{F}_{0}=\left\{u \in \mathbb{N}^{n+2} \mid u_{1}+\cdots+u_{n+1}=d u_{0}\right\}
$$

Maintenant pour tout $b \in \mathbb{R}, b>0$ et $c \in \mathbb{R}$ posons

$$
\begin{aligned}
L(b) & =\left\{\sum_{v \in \mathcal{F}_{0}} C_{v} X^{v} \mid C_{v} \in \Omega \text { et } \inf _{v}\left\{\operatorname{ord} C_{v}-b v_{0}\right\}>-\infty\right\}, \\
L^{*}(b) & =\left\{\sum_{v \in \mathcal{F}_{0}} C_{v} \frac{1}{X^{v}} \mid C_{v} \in \Omega \text { et } \inf _{v}\left\{\operatorname{ord} C_{v}+b v_{0}\right\}>-\infty\right\}, \\
L_{c}(b) & =\left\{\sum_{v \in \mathcal{F}_{0}} C_{v} X^{v} \mid C_{v} \in \Omega \text { et ord } C_{v}-b v_{0} \rightarrow+\infty\right\}, \\
L(b, c) & =\left\{\sum_{v \in \mathcal{F}_{0}} C_{v} X^{v} \mid C_{v} \in \Omega \text { et } \text { ord } C_{v} \geqslant b v_{0}+c\right\}, \\
M(b) & =\left\{1+\beta_{1} z+\beta_{2} z^{2}+\cdots \mid \beta_{j} \geqslant j b \quad \forall j \in \mathbb{N}^{*}\right\},
\end{aligned}
$$

où $\Omega$ est le complété de $\Omega_{0}$ par rapport à la norme dont on a muni $\Omega_{0}$ en 1.1. On observe que si $\xi^{*} \in L^{*}\left(b^{\prime}\right), \xi \in L(b)$ et $b>b^{\prime}$, alors $\gamma_{-}\left(\xi^{*} \cdot \xi\right)$ est bien défini et appartient à $L^{*}\left(b^{\prime}\right)$ et que si $b^{\prime}<b$ alors $L^{*}\left(b^{\prime}\right) \hookrightarrow L^{*}(b)$. Grâce à ces espaces on peut finalement commencer l'étude de $\mathcal{K}_{A}^{(l)}$.

THÉoRÈme 1.7. $\mathcal{K}_{A}^{(l)} \subset L^{*}(\varepsilon)$ pour tout $l \in \mathbb{N}-\{0\}$ et $\varepsilon>0$.

Preuve: Soit $R(A)$ le résultant de $h, h_{1}, \ldots, h_{n}$. Puisque d'après $1.2 R(A) \neq 0$, on peut écrire, grâce à [5, Théorème 6.2], les éléments d'une base de $\mathcal{K}_{A}^{(l)}$ de la façon suivante:

$$
w_{u, i, A}^{*}=\sum_{w \in \mathcal{F}_{0}} \frac{1}{\chi(A)} \frac{G_{w, u, i}(A)}{\left(R(A) \pi_{v}\right)^{w_{0}}} \frac{1}{X^{w}}
$$

où $G_{w, u, i}(A), \chi(A) \in \mathcal{O}_{\Omega_{0}}$. Mais alors grâce à 1.2 on observe que $\mathcal{K}_{A}^{(l)} \subset L^{*}(1 /(p-1))$. Maintenant pour démontrer qu'en réalité $\mathcal{K}_{A}^{(l)} \subset L^{*}(\varepsilon) \quad \forall \varepsilon>0$, il y a deux méthodes:

(1) la première, qu'on va appeler itération de Frobenius, est valide seulement pour $p \geqslant 3$;

(2) la deuxième est valide pour chaque $p$, mais elle est beaucoup plus compliquée. 
Avant de commencer l'exposition de l'itération de Frobenius, nous allons définir deux applications utiles dans ces deux méthodes:

$$
\begin{aligned}
& \phi: X^{v} \longmapsto X^{p v} \\
& \psi: X^{v} \longrightarrow \begin{cases}X^{v / p} & \text { si } p \mid v_{i} \quad \forall i=1, \ldots, n+1, \\
0 & \text { autrement. }\end{cases}
\end{aligned}
$$

(1) Commençons par l'itération de Frobenius.

Considérons l'application

$$
\theta: \mathcal{K}_{A^{p}}^{(l)} \longrightarrow L^{*}\left(\frac{1}{p(p-1)}\right)
$$

où $\theta=\gamma_{-} \circ G(A, X) \circ \phi$, avec $G(A, X)=\exp \left(\pi\left(X_{0} h(A, X)-X_{0}^{p} h\left(A^{p}, X^{p}\right)\right)\right)$. Puisque d'après de $[6,21.1] G(A, X) \in L\left((p-1) / p^{2}\right)$, on a que $\theta$ est bien définie si $p \neq$ 2. De plus si $D_{i, A^{p}}^{*}=\gamma_{-} \circ\left(-E_{i}+\pi X_{0} h_{i}\left(A^{p}, X\right)\right)$, on trouve que $D_{i, A}^{*} \circ \theta=p \theta \circ$ $D_{i, A^{p}}^{*}$ et par conséquent l'image de $\mathcal{K}_{A^{p}}^{(l)}$ par $\theta$ est contenue dans $\mathcal{K}_{A}^{(l)}$, c'est-à-dire, $\theta: \mathcal{K}_{A^{p}}^{(l)} \longrightarrow \mathcal{K}_{A}^{(l)} \otimes \Omega$. Mais alors, puisque $\theta$ est injective si $p \neq 2\left(\gamma_{-} \circ \psi \circ G(A, X)^{-1}\right.$ est un inverse à gauche sur l'image), grâce à (1.4.3) on obtient que $\mathcal{K}_{A}^{(l)} \subset L^{*}(1 /(p(p-1)))$ si $p \neq 2$. Maintenant en prenant comme point de départ cette dernière inclusion et en itérant ce processus on peut conclure que si $p \neq 2, \mathcal{K}_{A}^{(l)} \subset L^{*}(\varepsilon)$ pour tout $\varepsilon>0$.

(2) Pour démontrer la deuxième méthode qui est valide pour chaque $p$, on va utiliser "the $s=\infty$ splitting theory" que Dwork a introduit dans [3, Section 4] et [4, Section 3]. Comme cette méthode est très longue, on va la diviser en 5 parties:

I. Théorie à l'infini: l'espace $\mathcal{K}_{A, \infty}^{(l)}$.

Considérons la série formelle $s(t)=t+t^{p} / p+t^{p^{2}} / p^{2}+\cdots$. D'après [8, II 2.1] elle admet $p-1$ zéros d'ordre $1 /(p-1)$ : soit $\gamma$ le zéro qui est le plus proche de $\pi$. Pour simplifier les notations posons $\gamma_{l}=\sum_{s=0}^{l} \gamma^{p^{s}} / p^{s}$. On observe facilement que

$$
\operatorname{ord} \gamma_{l} \geqslant\left(p^{l+1} /(p-1)\right)-(l+1) .
$$

Soit $E(t)=\exp \left(\sum_{j=0}^{\infty}\left(t^{p^{j}} / p^{j}\right)\right)$ la série de Artin-Hasse. Avec cette terminologie définissons les fonctions

$$
\begin{gathered}
\theta(t)=E(\gamma t), \quad \hat{\theta}(t)=\prod_{j=0}^{\infty} \theta\left(t^{p^{j}}\right), \\
\widehat{G}_{\infty}(A, X)=\prod_{u \in \mathcal{F}} \hat{\theta}\left(X_{0} A_{u} X^{u}\right) .
\end{gathered}
$$


Soit maintenant $F=\sum_{l=0}^{\infty} \gamma_{l} X_{0}^{p^{l}} h\left(A^{p^{l}}, X^{p^{l}}\right)$ et pour $i=1, \ldots, n+1$, définissons les opérateurs diférentiels

$$
\begin{aligned}
& D_{A, i, \infty}=E_{i}+F_{i}, \\
& D_{A, i, \infty}^{*}=\gamma_{-} \circ\left(-E_{i}+F_{i}\right),
\end{aligned}
$$

où $F_{i}=E_{i} F$ pour $i=1, \ldots, n+1$. Le symbole $\infty$ sert à nous rappeler que $F$ est associé à $\widehat{G}_{\infty}(A, X) \operatorname{car} \exp F=\widehat{G}_{\infty}(A, X)$. Puisque d'après (1.7.1) $F_{i} \in L(p /(p-1),-1)$, on observe que si $0<b<p /(p-1)$, alors l'applications $D_{A, i, \infty}^{*}: L^{*}(b / p) \longrightarrow L^{*}(b / p)$ est bien définie et par conséquent pour $0<b<p /(p-1)$ et $l \in \mathbb{N}-\{0\}$, on peut définir les modules

$$
\mathcal{K}_{A, \infty}^{(l)}(b)=\left\{\xi^{*} \in L^{*}(b) \mid D_{A, \infty}^{* w} \xi^{*}=0 \quad \forall w \in \mathbb{N}^{n+1} \text { tels que }|w|=l\right\} .
$$

II. Si $1 /(p-1)<b<p /(p-1), \operatorname{dim}_{\Omega} \mathcal{K}_{A, \infty}^{(l)}(b)=\bar{N}$.

Avant de vérifier II, modifions la terminologie utilisée jusqu'ici. En effet pour tout $v=\left(v_{0}, \ldots, v_{n}\right) \in \mathbb{N}^{n+1}$, définissons $D_{A, \infty}^{v}=D_{A, 0, \infty}^{v_{0}} \cdots D_{A, n, \infty}^{v_{n}}$ (respectivement $D_{A, 1}^{v}=D_{A, 0,1}^{v_{0}} \cdots D_{A, n, 1}^{v_{n}}$ ), où pour $i=1, \ldots, n$, les $D_{A, i, \infty}$ (respectivement $D_{A, i, 1}$ ) sont les opérateurs définis en (1.7.3) (respectivement définis comme en (1.4.1) mais où on remplace $X_{j}$ par $\left.X_{j}\left(\pi_{v} X_{0}\right)^{1 \backslash d}\right)$ et

$$
D_{A, 0, \infty}=X_{0} \frac{\partial}{\partial X_{0}}+F \quad\left(\text { respectivement } D_{A, 0,1}=X_{0} \frac{\partial}{\partial X_{0}}+\pi X_{0} h(A, X)\right) .
$$

L'opérateur adjoint de $D_{A, 0, \infty}$ (respectivement $\left.D_{A, 0,1}\right)$ est $D_{A, 0, \infty}^{*}=\gamma_{-} \circ\left(-X_{0} \frac{\partial}{\partial X_{0}}\right.$ $+F)$ (respectivement $D_{A, 0,1}^{*}=\gamma_{-} \circ\left(-X_{0} \frac{\partial}{\partial X_{0}}+\pi X_{0} h(A, X)\right)$ ). Ce changement n'est pas très grave car si on considère les $D_{A, i, \infty}$ (respectivement $D_{A, i, 1}$ ) comme opérateurs de $R_{A}$, alors $D_{A, 0, \infty}=D_{A, 1, \infty}+\cdots+D_{A, n+1, \infty}$ (respectivement $D_{A, 0,1}=$ $\left.D_{A, 1,1}+\cdots+D_{A, n+1,1}\right)$, c'est-à-dire, du point de vue des espaces vectoriels, il n'y a pas de différence si on considère $D_{A, 0, \infty}, \ldots, D_{A, n, \infty}$ (respectivement $D_{A, 0,1}, \ldots, D_{A, n, 1}$ ) à la place de $D_{A, 1, \infty}, \ldots, D_{A, n+1, \infty}$ (respectivement $D_{A, 1,1}, \ldots, D_{A, n+1,1}$ ). Mais on doit se rappeler que 1.2 est vraie pour $R\left(h, \ldots, h_{n}\right)$ et non pour $R\left(h_{1}, \ldots, h_{n+1}\right)$. Commençons maintenant la démonstration de II. D'après [3, Lemme 3.6] il existe un sous espace $W_{\infty}$ de $\Omega[X]$, contenu dans $L(b)$, tel que si $1 /(p-1) \leqslant b \leqslant p /(p-1)$

$$
L(b)=W_{\infty}^{(l)}+\sum_{|v|=l} D_{A, \infty}^{v} L(b)
$$


où $W_{\infty}^{(l)}=W_{\infty}+\sum_{|v|=1} D_{A, \infty}^{v} W_{\infty}+\sum_{|v|=2} D_{A, \infty}^{v} W_{\infty}+\cdots+\sum_{|v|=l-1} D_{A, \infty}^{v} W_{\infty}$. Maintenant si on refait les mêmes calculs qu'a faits Dwork dans [5, Lemmes 2.1, 2.2 et Théorème 2.1], en remplaçant [5, Lemme 1.3] avec [3, Lemme 3.10], on trouve que si $1 /(p-1)<$ $b \leqslant p /(p-1)$

$$
W_{\infty}^{(l)} \bigcap \sum_{|v|=l} D_{A, \infty}^{v} L(b)=\{0\}
$$

$$
\operatorname{dim}_{\Omega} W_{\infty}^{(l)}=\bar{N}
$$

Puisque si $b^{\prime}<b$, les éléments de l'espace $L^{*}\left(b^{\prime}\right)$ agissent comme formes linéaires sur l'espace $L(b)$, on a que $\mathcal{K}_{A, \infty}^{(l)}\left(b^{\prime}\right)$ s'identifie à un sous espace de $\operatorname{Hom}(L(b) /$ $\left.\sum_{|w|=l} D_{A, \infty}^{w} L(b), \Omega\right)$ et donc grâce à (1.7.4), (1.7.5) et (1.7.6) on a que si $1 /(p-1)<$ $b^{\prime} \leqslant p /(p-1)$

$$
\operatorname{dim}_{\Omega} \mathcal{K}_{A, \infty}^{(l)}\left(b^{\prime}\right) \leqslant \bar{N}
$$

c'est-à-dire, la fonction

$$
\begin{aligned}
\left(\frac{1}{p-1} ; \frac{p}{p-1}\right] & \longrightarrow \mathbb{N} \\
b & \longmapsto \operatorname{dim}_{\Omega} \mathcal{K}_{A, \infty}^{(l)}(b)
\end{aligned}
$$

est majorée par $\bar{N}$. Par conséquent puisqu'elle est bornée et monotone croissante, elle est localement constante dans le complémentaire d'un ensemble fini $S$. Fixons un $b \in(1 /(p-1) ; p /(p-1)] \backslash S$. Il existe alors un $b^{\prime}<b$ tel que $\mathcal{K}_{A, \infty}^{(l)}(b) \subset L^{*}\left(b^{\prime}\right)$ et donc l'application

$$
\begin{aligned}
\mathcal{K}_{A, \infty}^{(l)}(b) \times L(b) & \longrightarrow \Omega \\
\left(\xi^{*}, \xi\right) & \longmapsto \text { coefficient de } X^{0} \text { dans le produit } \xi \cdot \xi^{*}
\end{aligned}
$$

est bien définie. D'autre part puisque pour tout $b \in \mathbb{R}, b>0, W_{\infty} \subset L_{c}(b)$ et d'après (1.7.1) $F$ et $F_{i} \in L(p /(p-1))$ pour $i=1, \ldots, n$, on observe que si $b<p /(p-1)$, $W_{\infty}^{(l)} \subset L_{c}(b)$. Donc si on définit $E(b)=L_{c}(b) \cap \sum_{|v|=l} D_{A, \infty}^{v} L(b)$, d'après (1.7.4), (1.7.5) et (1.7.6) on obtient que si $1 /(p-1)<b<p /(p-1)$

$$
\operatorname{dim}_{\Omega} L_{\mathrm{c}}(b) / E(b)=\bar{N} .
$$


Considérons maintenant les projections $\pi_{b}: L(b) \longrightarrow \mathcal{W}_{\infty}^{(l)}$ et $\pi_{c, b}: L_{c}(b) \longrightarrow$ $L_{c}(b) / E(b)$. Grâce à (1.7.5) elles sont bien définies. Vérifions que $\pi_{c, b}$ est continue: en itérant [3, Lemme 3.6], il existe un sous espace $V(b, c)$ de $\Omega(X)$ tel que si $1 /(p-1) \leqslant b \leqslant p /(p-1)$ et $e=b-(1 / p-1)$

$$
\begin{array}{r}
L(b, c)=V(b, c)+\sum_{|v|=1} D_{A, \infty}^{v} V(b, c+e)+\sum_{|v|=2} D_{A, \infty}^{v} V(b, c+2 e)+\cdots \\
\cdots+\sum_{|v|=l-1} D_{A, \infty}^{v} V(b, c+(l-1) e)+\sum_{|v|=l} D_{A, \infty}^{v} L(b, c+l e) .
\end{array}
$$

Ceci entraine que $\pi_{b}$ est continue et en particulier que son noyau, $\sum_{|v|=l} D_{A, \infty}^{v} L(b)$, est fermé dans $L(b)$. Par conséquent $E(b)$ est fermé dans $L_{c}(b)$ et donc $\pi_{c, b}$ est continue. Alors si $\chi$ est un élément de $\operatorname{Hom}\left(L_{c}(b) / E(b), \Omega\right), \chi \circ \pi_{c, b}$ est une fonction linéaire et continue sur $L_{c}(b)$. Mais puisque d'après [11] l'espace des fonctions linéaires et continues sur $L_{c}(b)$ est isomorphe à $L^{*}(b)$, il existe un $\xi^{*} \in L^{*}(b)$ tel que pour tout $\xi \in L_{c}(b)$

$$
\left(\chi \circ \pi_{c, b}\right)(\xi)=\left\langle\xi^{*}, \xi\right\rangle
$$

D'autre part $\pi_{c, b}$ annule $\sum_{|v|=l} D_{A, \infty}^{v} L_{c}(b)$, et donc en réalité $\xi^{*} \in \mathcal{K}_{A, \infty}^{(l)}(b)$. En résumant, on a construit l'application

$$
\begin{aligned}
\operatorname{Hom}\left(L_{c}(b) / E(b), \Omega\right) & \longrightarrow \mathcal{K}_{A, \infty}^{(l)}(b) \\
\chi & \longmapsto \xi^{*}
\end{aligned}
$$

qui est injective d'après (1.7.9). Alors grâce à (1.7.8) et (1.7.7) on obtient que $\operatorname{dim}_{\Omega} \mathcal{K}_{A, \infty}^{(l)}(b)=\bar{N}$ si $b \notin S$ et $1 /(p-1)<b<p /(p-1)$. Par conséquent puisque l'application $b \longmapsto \operatorname{dim}_{\Omega} \mathcal{K}_{A, \infty}^{(l)}(b)$ est croissante, majorée par $\bar{N}$ et atteint son maximum sur le complémentaire d'un ensemble fini, elle est constante, c'est-à-dire, si $1 /(p-1)<b<p /(p-1), \operatorname{dim}_{\Omega} \mathcal{K}_{A, \infty}^{(l)}(b)=\bar{N}$.

III. Si $0<b<p /(p-1), \operatorname{dim}_{\Omega} \mathcal{K}_{A, \infty}^{(l)}(b)=\bar{N}$. Posons $G(A, X)=\prod_{u \in \mathcal{F}} \theta\left(X_{0} A_{u} X^{u}\right)$ et considérons l'application

$$
\alpha^{*}: L^{*}(b) \longrightarrow L^{*}\left(\frac{b}{p}\right)
$$

où $\alpha^{*}=\gamma_{-} \circ G(A, X) \circ \phi$. Puisque d'après [6, 21.1] $G(A, X) \subset L(1 /(p-1))$, on a que si $0<b<p /(p-1)$, $\alpha^{*}$ est bien défnie et injective $\left(\gamma_{-} \circ \psi \circ G(A, X)^{-1}\right.$ est 
son inverse à gauche sur l'image). Mais alors aussi $\alpha_{s}^{*}: L^{*}(b) \longrightarrow L^{*}\left(b / p^{s}\right)$, qui est l's-ième itération de $\alpha^{*}$, est bien définie et injective si $0<b<p /(p-1)$. D'autre part puisque $D_{A, i, \infty}^{*} \circ \alpha^{*}=p \alpha^{*} \circ D_{A{ }^{p}, i, \infty}^{*}$, on a que $\alpha_{s}^{*}\left(\mathcal{K}_{A^{p}{ }^{s}, \infty}^{(l)}(b)\right) \subset \mathcal{K}_{A, \infty}^{(l)}\left(b / p^{s}\right)$, et donc grâce à II on obtient que $\bar{N} \leqslant \operatorname{dim}_{\Omega} \mathcal{K}_{A, \infty}^{(l)}\left(b / p^{s}\right)$ si $1 /(p-1)<b<p /(p-1)$. Mais puisque $\mathcal{K}_{A, \infty}^{(l)}\left(b / p^{s}\right)$ s'injecte dans $\mathcal{K}_{A, \infty}^{(l)}(b)$, toujours grâce à II on trouve l'inégalité inverse et par conséquent on peut finalement conclure que si $0<b^{\prime}<p /(p-1)$, $\operatorname{dim}_{\Omega} \mathcal{K}_{A, \infty}^{(l)}\left(b^{\prime}\right)=\bar{N}$

IV. Notre théorie: l'espace $\mathcal{K}_{A, 1}^{(l)}$.

Posons

$$
\begin{gathered}
\theta_{1}(t)=\exp \pi\left(t-t^{p}\right), \quad \widehat{\theta}_{1}(t)=\prod_{j=0}^{\infty} \theta_{1}\left(t^{p^{j}}\right), \\
\widehat{G}_{1}(A, X)=\prod_{u \in \mathcal{F}} \hat{\theta}_{1}\left(X_{0} A_{u} X^{u}\right),
\end{gathered}
$$

et pour $i=1, \ldots, n+1$, définissons les opérateurs différentiels

$$
\begin{aligned}
& D_{A, i, 1}=E_{i}+\pi X_{0} h_{i}(A, X), \\
& D_{A, i, 1}^{*}=\gamma_{-} \circ\left(-E_{i}+\pi X_{0} h_{i}(A, X)\right) .
\end{aligned}
$$

L'indice 1 sert à nous rappeler que $\pi X_{0} h(A, X)$ est associé à $\widehat{G}_{1}(A, X)$ puisque $\widehat{G}_{1}(A, X)=\exp \left(\pi X_{0} h(A, X)\right)$. On remarque que ces opérateurs sont comme ceux définis en (1.4.1) et (1.5.1) mais où on remplace $X_{j}$ par $X_{j}\left(\pi_{v} X_{0}\right)^{1 \backslash d}$, et donc pour chaque $b \in \mathbb{R}, b>0, \mathcal{K}_{A, 1}^{(l)}(b)=\mathcal{K}_{A}^{(l)} \cap L^{*}(b)$, où $\mathcal{K}_{A}^{(l)}$ est le module introduit en 1.5.

V. Transition entre notre théorie et la théorie à l'infini. Introduisons le quotient $k(t)=\frac{\widehat{\theta}_{1}(t)}{\widehat{\theta}(t)}$ des fonctions $\widehat{\theta}(t)$ et $\widehat{\theta}_{1}(t)$ définies respectivement en (1.7.2) et (1.7.10). Vérifions que $k(t) \in M((p-1) / p)$ : puisque $k(t)=$ $\exp (t(\pi-\gamma)) \cdot \prod_{l=1}^{\infty} \exp \left(-\pi t^{p^{l}} \gamma_{l}\right)$ il suffit de démontrer que pour tout $l \geqslant 1, \exp \left(-\pi t^{p^{l}} \gamma_{l}\right)$ et $\exp (t(\pi-\gamma)) \in M((p-1) / p)$. Grâce à (1.7.1) on montre très facilement que pour chaque $l \geqslant 1, \exp \left(-\pi t^{p^{l}} \gamma_{l}\right) \in M((p-1) / p)$. Pour vérifier que $\exp (t(\pi-\gamma)) \in$ $M((p-1) / p)$, posons $\gamma=\pi z$ pour un certain $z$. Puisque $s(\gamma)=0$ on a

$$
h(z) \stackrel{\text { déf }}{=} 1+\frac{(z \pi)^{p-1}}{p}+\frac{(z \pi)^{p^{2}-1}}{p^{2}}+\cdots=0 .
$$

On observe que $h(z)$ est analytique et limité par 1 dans le disque $D\left(0,1^{-}\right)$et donc $\forall z \in D\left(1,1^{-}\right)$

$$
h(z)=B_{0}+B_{1}(z-1)+B_{2}(z-1)^{2}+\ldots
$$


avec $B_{s}=\left(h^{(s)}(1)\right) /(s !),\left|B_{0}\right| \leqslant|p|,\left|B_{1}\right|=1$ et $\left|B_{s}\right| \leqslant 1$ pour chaque $s \in \mathbb{N}$. Par conséquent d'après $[8$, II 2.1$]$ il existe un seul zéro $z_{0}$ de $h(z)$ tel que $\left|1-z_{0}\right| \leqslant|p|$. Mais alors, puisqu'au début de I on a choisit $\gamma$ comme étant le zéro le plus proche de $\pi$, on a $\gamma=z_{0} \pi$ et donc on trouve que $\exp (t(\pi-\gamma)) \in M((p-1) / p)$.

Maintenant soit $\mathcal{G}=\prod_{u \in \mathcal{F}} k\left(X_{0} A_{u} X^{u}\right)$. Puisque $k(t) \in M((p-1) / p)$, si $0<b<$ $((p-1) / p)$, l'application

$$
\gamma_{-} \circ \mathcal{G}: L^{*}(b) \longrightarrow L^{*}(b)
$$

est bien définie et est un isomorphisme ( $\gamma_{-} \circ \mathcal{G}^{-1}$ est son inverse à droite et à gauche). D'autre part puisque $D_{A, i, 1}^{*} \circ \gamma_{-} \circ \mathcal{G}=\gamma_{-} \circ \mathcal{G} \circ D_{A, i, \infty}^{*}$, on trouve que $\gamma_{-} \circ \mathcal{G}\left(\mathcal{K}_{A, \infty}^{(l)}(b)\right) \subseteq$ $\mathcal{K}_{A, 1}^{(l)}(b) \otimes \Omega$ et donc grâce à III et (1.4.3) on peut enfin conclure que si $0<b<$ $((p-1) / p), \mathcal{K}_{A}^{(l)}=\mathcal{K}_{A, 1}^{(l)}(b)$.

Corollatre 1.8. Soit

$$
\left\{w_{u, i, A}^{*}=\sum_{w \in \mathcal{F}_{0}} \frac{1}{\chi(A)} \frac{G_{w, u, i}(A)}{(R(A) \pi)^{w_{0}}} \frac{1}{X^{w}}\right\}_{\substack{1 \leqslant i \leqslant N \\ 1 \leqslant|u| \leqslant l-1}}
$$

une base de $\mathcal{K}_{A}^{(l)}$. Alors

$$
\operatorname{ord}_{A} \frac{G_{w, u, i}(A)}{\chi(A) \pi^{w_{0}}} \geqslant-w_{0} \varepsilon+o(1) \quad \forall \varepsilon>0 .
$$

\section{CAS D'UNE SUITE RÉGULIÈRE}

2.0. Dans ce paragraphe on va démontrer, sous l'hypothèse de régularité, que $\mathcal{W}^{(l)}$ est un module différentiel de type $G$. Commençons avec le cas $l=1$

Proposition 2.1. Si $f_{1}, \ldots, f_{n+1}$ est une suite régulière, alors $\operatorname{dim}_{K(\lambda)} \mathcal{W}^{(1)}$ $=N$. Par conséquent $\mathcal{W}^{(1)}$, muni de la connexion $\sigma_{\lambda}$, est un module différentiel sur $K(\lambda)$.

Preuve: Conséquence de la décomposition de l'espace $R$ qui se trouve dans [5, Section 1].

2.2. Maintenant on va spécialiser la théorie duale introduite en 1.5:

$$
\begin{array}{rlr}
R_{\lambda}^{*} & =\operatorname{Hom}(R, K(\lambda)), \\
D_{i, \lambda}^{*} & =\gamma_{-} \circ\left(-E_{i}+f_{i}(\lambda, X)\right) & (1 \leqslant i \leqslant n+1), \\
\mathcal{K}_{\lambda}^{(1)} & =\left\{\xi^{*} \in R_{\lambda}^{*} \mid D_{i, \lambda}^{*} \xi^{*}=0\right. & \forall i=1, \ldots, n+1\} .
\end{array}
$$

De plus on modifie aussi les espaces $L(b)$ et $L^{*}(b)$, introduits en 1.6 , en remplaçant $\Omega$ par $\Omega_{v}$, un corps qui soit complet pour une extension de la valeur absolue $v$-adique de 
$K\left(\pi_{v}\right)$ et qui contienne un point générique $t_{v}$. On considère $\lambda$ comme une variable de $\Omega_{v}$. Grâce à ces espaces et à la théorie duale on peut finalement démontrer que

$$
\rho(G(\lambda))=\sum_{v \in \mathcal{P}_{f}} \log ^{+} \frac{1}{R_{v}}<+\infty
$$

où $G(\lambda)$ est une matrice $N \times N$ qui représente la connexion $\sigma_{\lambda}$ sur $\mathcal{W}^{(1)}$ et $R_{v}$ est le rayon de convergence de la matrice solution du système $\frac{\partial}{\partial \lambda}-G(\lambda)=0$ au point générique $t_{v}$.

THÉORÈME 2.3. Si $f_{1}, \ldots, f_{n+1}$ est une suite régulière, alors pour chaque $v \in$ $\mathcal{P}_{f}$ le rayon de convergence, $R_{v}$, de la matrice solution de $\frac{\partial}{\partial \lambda}-G(\lambda)=0$ au point générique $t_{v}$, est au moins $\left|R\left(t_{v}\right)\right|$. Par conséquent

$$
\rho(G(\lambda)) \leqslant \sum_{v \in \mathcal{P}_{f}} \log \frac{1}{|R(\lambda)|_{v}} .
$$

PREUVE: Simplifions les notations en indiquant avec $R(\lambda)$ (respectivement $R\left(t_{v}\right)$ ) le résultant des polynômes $f_{1}(\lambda, X), \ldots, f_{n+1}(\lambda, X)$ (respectivement $f_{1}\left(t_{v}, X\right), \ldots$, $\left.f_{n+1}\left(t_{v}, X\right)\right)$. Pour trouver la matrice solution au point générique $t_{v}$ du système $\frac{\partial}{\partial \lambda}-G(\lambda)=0$, considérons l'application

$$
T_{t_{v}, \lambda}^{*}: \mathcal{K}_{t_{v}} \longrightarrow \mathcal{K}_{\lambda} \otimes \Omega_{v}
$$

où $T_{t_{v}, \lambda}^{*}=\gamma_{-} \circ \exp \pi X_{0}\left(f(X, \lambda)-f\left(X, t_{v}\right)\right)$. Vérifions si cette application est bien définie. En faisant des calculs très simples, qu'on laisse au lecteur, on trouve

$$
\exp \pi X_{0}\left(f(X, \lambda)-f\left(X, t_{v}\right)\right) \in L\left(\operatorname{ord}\left(\lambda-t_{v}\right)\right) .
$$

D'autre part en spécialisant de façon convenable le vecteur $A$ de 1.8 on obtient

$$
\mathcal{K}_{t_{v}}^{(1)} \subset L^{*}\left(\operatorname{ord} R\left(t_{v}\right)\right)
$$

Mais alors pour tout $\xi^{*} \in \mathcal{K}_{t_{v}}$, le produit $T_{t_{v}, \lambda}^{*}\left(\xi^{*}\right)$ existe si $\operatorname{ord}\left(\lambda-t_{v}\right)>\operatorname{ord} R\left(t_{v}\right)$. De plus puisque $T_{t_{v}, \lambda}^{*} \circ D_{i, t_{v}}^{*}=D_{i, \lambda}^{*} \circ T_{t_{v}, \lambda}^{*}$, on a que si $\xi^{*} \in \mathcal{K}_{t_{v}}$ alors $T_{t_{v}, \lambda}^{*}\left(\xi^{*}\right) \in \mathcal{K}_{\lambda} \otimes \Omega_{v}$. On peut donc conclure que $T_{t_{v}, \lambda}^{*}$ est bien définie si $\left|\lambda-t_{v}\right|<\left|R\left(t_{v}\right)\right|$.

Maintenant soit $\left\{w_{i, \lambda}^{*}\right\}_{1 \leqslant i \leqslant N}$ (respectivement $\left\{w_{i, t_{v}}^{*}\right\}_{1 \leqslant i \leqslant N}$ ) une base de $\mathcal{K}_{\lambda}$ (respectivement de $\mathcal{K}_{t_{v}}$ ) qui a pour base duale la base $\left\{w_{i, \lambda}\right\}_{1 \leqslant i \leqslant N}$ (respectivement $\left.\left\{w_{i, t_{v}}\right\}_{1 \leqslant i \leqslant N}\right)$ composée de monômes de la forme $\pi_{v}^{u_{0}} X^{u}$ avec $u \in \mathcal{F}_{0}$. De plus 
soient $\sigma_{\lambda}^{*}=\gamma_{-} \circ\left(\frac{\partial}{\partial \lambda}-\frac{\partial f}{\partial \lambda}\right)$ et $\mathcal{Y}\left(t_{v}, \lambda\right)^{t}$ la matrice de l'application $T_{t_{v}, \lambda}^{*}$. Puisque $T_{t_{v}, \lambda}^{*} \circ \frac{\partial}{\partial \lambda}=\sigma_{\lambda} \diamond T_{t_{v}, \lambda}^{*}$, on observe que

$$
\frac{\partial}{\partial \lambda} \mathcal{Y}\left(t_{v}, \lambda\right)=G(\lambda) \mathcal{Y}\left(t_{v}, \lambda\right)
$$

c'est-à-dire, $\mathcal{Y}\left(t_{v}, \lambda\right)$ est la matrice solution au point générique $t_{v}$ dont on doit chercher le rayon de convergence $R_{v}$. Mais alors, si $w_{i, t_{v}}^{*}=\sum_{u \in \mathcal{F}_{0}} B_{u}\left(1 / X^{u}\right)$ et $T_{t_{v}, \lambda}^{*}=\gamma_{-}$。 $\left(\sum_{w \in \mathcal{F}_{0}} H_{w} X^{w}\right)$, grâce à (2.3.1) et (2.3.2) on a

$$
\mathcal{Y}\left(t_{v}, \lambda\right)_{j, i}=\left\langle T_{t_{v}, \lambda}^{*}\left(w_{i, t_{v}}^{*}\right), \pi_{v}^{u_{0}} X^{u}\right\rangle=\sum_{w \in \mathcal{F}_{0}} H_{w} B_{w+u}
$$

avec

$$
\operatorname{ord}\left(H_{w} B_{w+u}\right) \geqslant w_{0}\left(\operatorname{ord}\left(\lambda-t_{v}\right)-\left(\operatorname{ord} R\left(t_{v}\right)\right)\right)-u_{0} \operatorname{ord} R\left(t_{v}\right)+o(1)
$$

et donc on peut conclure que $R_{v} \geqslant\left|R\left(t_{v}\right)\right|$.

\subsection{Passons au cas $l>1$ :}

PROPOS ITI ION 2.5. Si $f_{1}, \ldots, f_{n+1}$ est une suite régulière, alors $\operatorname{dim}_{K(\lambda)} \mathcal{W}^{(l)}=$ $\bar{N}$. Par conséquent pour chaque $l \in \mathbb{N}, l>1, \mathcal{W}^{(l)}$, muni de la connexion $\sigma_{\lambda}$, est un module différentiel sur $K(\lambda)$.

Preuve: Conséquence de [5, Théorème 2.1].

PROPOS ITION 2.6. Si $f_{1}, \ldots, f_{n+1}$ est une suite régulière, alors pour chaque $l \in \mathbb{N}, l>1, \mathcal{W}^{(l)}$ est de type $G$.

Preuve: Soit $G(\lambda)$ la matrice $N \times N$ à coefficients dans $K(\lambda)$ qui représente la connexion $\sigma_{\lambda}$ sur le module de type $G, \mathcal{W}^{(1)}$, c'est-à-dire,

$$
\sigma_{\lambda}\left(\begin{array}{c}
w_{1} \\
\vdots \\
w_{N}
\end{array}\right)=G(\lambda)\left(\begin{array}{c}
w_{1} \\
\vdots \\
w_{N}
\end{array}\right)+\sum_{i \in\{1, \ldots, n+1\}} D_{i} R .
$$

Si on applique $D^{v}$, avec $|v| \leqslant l-1$, à droite et à gauche de cette dernière égalité, on obtient

$$
\sigma_{\lambda}\left(\begin{array}{c}
D^{v} w_{1} \\
\vdots \\
D^{v} w_{N}
\end{array}\right)=G(\lambda)\left(\begin{array}{c}
D^{v} w_{1} \\
\vdots \\
D^{v} w_{N}
\end{array}\right)+\sum_{|w|=1+|v|} D^{w} R .
$$


Par conséquent si on choisit comme base de $\mathcal{W}^{(l)}$, la base ordonnée

$$
\left\{w_{j}: 1 \leqslant j \leqslant N ; D^{v} w_{j}:|v|=1,1 \leqslant j \leqslant N ; \ldots ; D^{v} w_{j}:|v|=l-1,1 \leqslant j \leqslant N\right\},
$$

on trouve que la matrice $\mathcal{G}(\lambda)$ qui représente la connexion $\sigma_{\lambda}$ sur $\mathcal{W}^{(l)}$ est de la forme

$$
\left(\begin{array}{ccccc}
G(\lambda) & & & & \\
& G(\lambda) & & \star & \\
& & \ddots & & \\
& O & & \ddots & \\
& & & & G(\lambda)
\end{array}\right)
$$

où la partie triangulaire supérieure est composée de matrices $N \times N$ à coefficients dans $K(\lambda)$. Mais alors puisque $G(\lambda)$ est la matrice d'un module différentiel de type $G$, on peut conclure grâce à [1, IV 3.3 Lemma 2].

\section{CAS D'UNE SUITE NON RÉGULIÈRE}

3.0. Dans ce paragraphe on va démontrer que même sans l'hypothèse de régularité, $\mathcal{W}^{(l)}$ est pour tout $l \geqslant 1$ un module différentiel de type $G$. Pour traiter ce cas, on va introduire une nouvelle variable, soit $\Gamma$, et on va utiliser la notion de polynôme générique, introduite dans la Section 1 , de façon à nous réduire au cas d'une suite régulière.

3.1. Soit $g(\lambda, \Gamma, A, X)=f(\lambda, X)+\Gamma h(A, X)$ où $h(A, X)=\sum_{u \in \mathcal{F}} A_{u} X^{u}$ est un polynôme générique. Posons $g_{i}(\lambda, \Gamma, A, X)=E_{i} g(\lambda, \Gamma, A, X)$ pour $i=1, \ldots, n+1$. Puisque le résultant $R(\lambda, \Gamma, A)$ de $g_{1}(\lambda, \Gamma, A, X), \ldots, g_{n+1}(\lambda, \Gamma, A, X)$ est un élément de $\mathcal{O}_{K}[\lambda, \Gamma, A]$ qui s'annule quand $\Gamma=0$, on a

$$
R(\lambda, \Gamma, A)=\Gamma^{e}\left(\rho_{0}(\lambda, A)+\Gamma \rho_{1}(\lambda, A)+\Gamma^{2} \rho_{2}(\lambda, A)+\ldots\right)
$$

où $\rho_{0}(\lambda, A) \neq 0$ et pour chaque $i \in \mathbb{N}, \rho_{i}(\lambda, A)$ est un polynôme homogène de degré $e+i$ par rapport aux variables $\left\{A_{u}\right\}_{u \in \mathcal{F}}$. Maintenant spécialisons $A$ dans un $A^{(0)} \in$ $\mathcal{O}_{K}^{\left(\begin{array}{c}n+d \\ n\end{array}\right)}$ tel que $\rho_{0}\left(\lambda, A^{(0)}\right) \neq 0$. (Dans la suite, si c'est nécessaire, on va modifier cette spécialisation). Les polynômes $g_{1}\left(\lambda, \Gamma, A^{(0)}, X\right), \ldots, g_{n+1}\left(\lambda, \Gamma, A^{(0)}, X\right)$ forment alors une suite régulière, c'est-à-dire, $R\left(\lambda, \Gamma, A^{(0)}\right) \neq 0$. Pour simplifier les notations on pose

$$
g(\lambda, \Gamma, X) \stackrel{\text { def } f}{=} g\left(\lambda, \Gamma, A^{(0)}, X\right)=f(\lambda, X)+\Gamma h\left(A^{(0)}, X\right),
$$

$$
R(\lambda, \Gamma) \stackrel{\text { déf }}{=} R\left(\lambda, \Gamma, A^{(0)}\right)=\Gamma^{e}\left(\rho_{0}\left(\lambda, A^{(0)}\right)+\Gamma \rho_{1}\left(\lambda, A^{(0)}\right)+\Gamma^{2} \rho_{2}\left(\lambda, A^{(0)}\right)+\ldots\right) .
$$


REMARque 3.2. On peut expliquer ce qu'on a fait en 3.1 de la façon suivante: notre but est d'étudier $f(\lambda, X)$ en le rendant une spécialisation $(\Gamma=0)$ d'une famille qui est génériquement régulière $\{g(\lambda, \Gamma, A, X)\}_{\Gamma}$. Cependant il y a plusieurs choix pour cette famille: chaque fois qu'on spécialise $A$, on obtient une famille différente. Notre but est de choisir la famille, c'est-à-dire, la spécialisation de $A$, qui rend minimum l'entier "e" qui apparait dans l'équation (3.1.1).

3.3. Avant de commencer l'étude des modules' $\mathcal{W}^{(l)}$ sous l'hypothèse d'irrégularité, on va spécialiser les notions introduites en 1.4 et 1.5 pour le polynôme générique afin d'en trouver d'analogues pour le polynôme $g(\lambda, \Gamma, X)$. On obtient

$$
\begin{aligned}
R_{\lambda, \Gamma} & =\sum_{u \in \mathcal{F}} K(\lambda, \Gamma) X^{u}, \\
R_{\lambda, \Gamma}^{*} & =\operatorname{Hom}\left(R_{\lambda, \Gamma}, K(\lambda, \Gamma)\right), \\
D_{i, \lambda, \Gamma} & =E_{i}+g_{i}(\lambda, \Gamma, X) \quad(1 \leqslant i \leqslant n+1), \\
D_{i, \lambda, \Gamma}^{*} & =\gamma_{-} \circ\left(-E_{i}+g_{i}(\lambda, \Gamma, X)\right) \quad(1 \leqslant i \leqslant n+1), \\
\mathcal{W}_{\lambda, \Gamma}^{(l)} & =R_{\lambda, \Gamma} / \sum_{|w|=l} D_{\lambda, \Gamma}^{w} R_{\lambda, \Gamma}, \\
\mathcal{K}_{\lambda, \Gamma}^{(l)} & =\left\{\xi^{*} \in R_{\lambda, \Gamma}^{*}\left|D_{\lambda, \Gamma}^{* w} \xi^{*}=0 \quad \forall\right| w \mid=l\right\} .
\end{aligned}
$$

3.4. Commençons par démontrer que, même dans le cas d'une suite non régulière, $\mathcal{W}^{(l)}$ est un module différentiel pour tout $l \geqslant 1$ :

Proposition 3.5. Soit $f_{1}, \ldots, f_{n+1}$ une suite non régulière. Alors pour chaque $l \in \mathbb{N}-\{0\}, \mathcal{W}^{(l)}$ est un module différentiel sur $K(\lambda)$.

PreUve: Considérons l'application

$$
T_{0, \Gamma}^{*}: \mathcal{K}_{\lambda, 0}^{(l)} \longrightarrow \mathcal{K}_{\lambda, \Gamma}^{(l)} \otimes K(\lambda)((\Gamma))
$$

où $T_{0, \Gamma}^{*}=\gamma_{-} \circ \exp (g(\lambda, \Gamma, X)-g(\lambda, 0, X))$. Vérifions qu'elle est bien définie. Si $\xi^{*} \in$ $\mathcal{K}_{\lambda, 0}^{(l)}$ alors le produit $\exp \left(\Gamma h\left(A^{(0)}, X\right)\right) \cdot \xi^{*}$ est bien défini puisque $\xi^{*}=\sum_{u \in \mathcal{F}} C_{u}\left(1 / X^{u}\right)$ avec $C_{u}$ indépendant de $\Gamma$ et

$$
T_{0, \Gamma}^{*}=\gamma_{-} \circ \sum_{v \in \mathcal{F}} B_{v}(\lambda, \Gamma) X^{v}, \quad B_{v}(\lambda, \Gamma) \in K(\lambda)[[\Gamma]]
$$

avec $\lim _{v \rightarrow \infty} B_{v}(\lambda, \Gamma)=\infty$. De plus puisque $D_{i, \lambda, \Gamma}^{*} \circ T_{0, \Gamma}^{*}=T_{0, \Gamma}^{*} \circ D_{i, \lambda, 0}^{*}$, on a que si $\xi^{*} \in \mathcal{K}_{\lambda, 0}^{(l)}$ alors $T_{0, \Gamma}^{*}\left(\xi^{*}\right) \in \mathcal{K}_{\lambda, \Gamma}^{(l)} \otimes K(\lambda)((\Gamma))$. On peut donc conclure que $T_{0, \Gamma}^{*}$ est bien définie. 
Puisque $T_{0, \Gamma}^{*}$ est injective $\left(T_{\Gamma, 0}^{*}=\gamma_{-} \circ \exp (g(\lambda, 0, X)-g(\lambda, \Gamma, X))\right.$ est un inverse à gauche sur l'image) et puisque $\sigma_{\Gamma}^{*} \circ T_{0, \Gamma}^{*}=T_{0, \Gamma}^{*} \circ \frac{\partial}{\partial \Gamma}$ implique

$$
T_{0, \Gamma}^{*}\left(\mathcal{K}_{\lambda, 0}^{(l)}\right) \subset \operatorname{Ker}\left(\sigma_{\Gamma}^{*}, \mathcal{K}_{\lambda, \Gamma}^{(l)} \otimes K(\lambda)((\Gamma))\right) \text {, }
$$

on peut conclure grâce à $[8$, III 1.2$]$ et à 2.5 que

$$
\begin{aligned}
\operatorname{dim}_{K(\lambda)} \mathcal{K}_{\lambda, 0}^{(l)}=\operatorname{dim}_{K(\lambda)} T_{0, \Gamma}^{*}\left(\mathcal{K}_{\lambda, 0}^{(l)}\right) & \leqslant \operatorname{dim}_{K(\lambda)} \operatorname{Ker}\left(\sigma_{\Gamma}^{*}, \mathcal{K}_{\lambda, \Gamma}^{(l)} \otimes K(\lambda)((\Gamma))\right) \\
& \leqslant \operatorname{dim}_{K(\lambda)(\Gamma)} \mathcal{K}_{\lambda, \Gamma}^{(l)}=\bar{N}<+\infty .
\end{aligned}
$$

3.6. Maintenant, comme en 2.3, pour démontrer que $\mathcal{W}^{(l)}$ est de type $G$, on va vérifier que $\rho(\mathcal{G}(\lambda))<+\infty$, où $\mathcal{G}(\lambda)$ est la matrice qui représente la connexion $\sigma_{\lambda}$ sur $\mathcal{W}^{(l)}$. Commençons par chercher des informations sur les éléments de $\mathcal{K}_{\lambda, 0}^{(l)}$. On observe que si $w_{\lambda, 0}^{*}$ est un élément d'une base de $\mathcal{K}_{\lambda, 0}^{(l)}$ et si $T_{0, \Gamma}^{*}$ est l'application définie en 3.5, alors

$$
T_{0, \Gamma}^{*}\left(w_{\lambda, 0}^{*}\right)_{\mid \Gamma=0}=w_{\lambda, 0}^{*} .
$$

(Grâce à (3.5.1) on sait que l'on peut spécialiser le $\Gamma$ en 0 ). Donc, pour avoir des information sur $w_{\lambda, 0}^{*}$ il suffit d'étudier $T_{0, \Gamma}^{*}\left(w_{\lambda, 0}^{*}\right)$. Puisque $R(\lambda, \Gamma) \neq 0$, grâce à $\left[5\right.$, Théorème 2.1], on sait que si $\left\{w_{i, \lambda, \Gamma}\right\}_{1 \leqslant i \leqslant N}$ est une base de $\mathcal{W}_{\lambda, \Gamma}^{(1)}$ alors $\left\{D^{v} w_{i, \lambda, \Gamma}\right\}_{\substack{0 \leqslant|v| \leqslant l-1 \\ 1 \leqslant i \leqslant N}}$ est une base de $\mathcal{W}_{\lambda, \Gamma}^{(l)}$. En supposant que les $D^{v} w_{i, \lambda, \Gamma}$ soient des éléments de $K[\lambda, \Gamma, X]$, on obtient que

$$
\theta_{i, v}=\left\langle T_{0, \Gamma}^{*}\left(w_{\lambda, 0}^{*}\right), D^{v} w_{i, \lambda, \Gamma}\right\rangle
$$

est une combinaison $K[\lambda, \Gamma]$-linéaire de coefficients de $\exp \left(\Gamma h\left(A^{(0)}, X\right)\right) \cdot w_{\lambda, 0}^{*}$ et donc c'est un élément de $K(\lambda)[[\Gamma]]$, c'est-à-dire,

$$
T_{0, \Gamma}^{*}\left(w_{\lambda, 0}^{*}\right)=\sum_{i=1}^{N} \sum_{0 \leqslant|v| \leqslant l-1} \theta_{i, v}(\lambda, \Gamma) w_{i, v, \lambda, \Gamma}^{*}
$$

où les $\theta_{i, v}(\lambda, \Gamma)$ sont des éléments de $K(\lambda)[[\Gamma]]$ et les $w_{i, v, \lambda, \Gamma}^{*}$ sont des éléments de la base de $\mathcal{K}_{\lambda, \Gamma}^{(l)}$, qui est duale à la base $\left\{D^{v} w_{i, \lambda, \Gamma}\right\}_{\substack{0 \leqslant|v| \leqslant l-1 \\ 1 \leqslant i \leqslant N}}$. En réalité on peut avoir des informations plus précises sur le vecteur $\vec{\theta}=\left(\theta_{i, v}(\lambda, \Gamma)\right)_{i, v}$ : d'après (3.5.2) ce vecteur satisfait le système différentiel

$$
\frac{\partial}{\partial \Gamma}-\mathcal{G}(\lambda, \Gamma)=0
$$


où $\mathcal{G}(\lambda, \Gamma)$ est une matrice $\bar{N} \times \bar{N}$ à coefficients dans $K(\lambda, \Gamma)$ qui représente la connexion $\sigma_{\Gamma}=\frac{\partial}{\partial \Gamma}+\frac{\partial g}{\partial \Gamma} \operatorname{sur} \mathcal{W}_{\lambda, \Gamma}^{(l)}$

3.7. Dans le théorème suivant nous allons étudier plus en détail $\vec{\theta}$, mais avant, comme en 1.6, on va faire un petit changement de notations: pour simplifier les calculs on introduit $\pi_{v} X_{0}$, c'est-à-dire, on remplace $X_{i}$ par $X_{i}\left(\pi_{v} X_{0}\right)^{1 \backslash d}$.

3.8 NOTA BENE. Après ce changement, la connexion $\sigma_{\Gamma}$ devient $\sigma_{\Gamma}=\left(\frac{\partial}{\partial \Gamma}+\right.$ $\left.X_{0} \pi_{v} \frac{\partial g}{\partial \Gamma}\right)$, mais les coefficients de la matrice $\mathcal{G}(\lambda, \Gamma)$ appartiennent toujours à $K(\lambda, \Gamma)$ c'est-à-dire, le système différentiel $\frac{\partial}{\partial \Gamma}-\mathcal{G}(\lambda, \Gamma)=0$ ne dépend pas de $v$.

ThÉorème 3.9. Soit $v \in \mathcal{P}_{f}$. Munissons $K(\lambda, \Gamma)$ de la norme de Gauss par rapport à $\lambda$ et $\Gamma$, et soit $\Omega$ la clôture algébrique de $K(\lambda, \Gamma)$ munie d'une valeur absolue qui prolonge celle de $K(\lambda, \Gamma)$. De plus soit $\vec{\theta}^{t}=\left(\theta_{i, w}(\lambda, \Gamma)\right)_{\substack{0 \leqslant|w| \leqslant l-1 \\ 1 \leqslant i \leqslant N}}$ le vecteur à coefficients dans $K(\lambda)[[\Gamma]]$ tel que $\frac{\partial}{\partial \Gamma} \vec{\theta}=\mathcal{G}(\lambda, \Gamma) \vec{\theta}$ avec $\mathcal{G}(\lambda, \Gamma)$ définie comme en 3.6. Alors $\theta_{i, w}(\lambda, \Gamma)$ converge pour

$$
|\Gamma|<\left|\rho_{0}\left(\lambda, A^{(0)}\right)\right|^{1+N^{2}}
$$

Par conséquent, si $\theta_{i, w}(\lambda, \Gamma)=\sum_{s=0}^{\infty} h_{i, w, s} \Gamma^{s}$ avec $h_{i, w, s} \in K(\lambda)$ pour tout $s$, alors pour chaque $\varepsilon<1$, il existe $M(\varepsilon)>0$ tel que

$$
\left|h_{i, w, s}\right| \leqslant \frac{M(\varepsilon)}{\left(\varepsilon\left|\rho_{0}\left(\lambda, A^{(0)}\right)\right|\right)^{s\left(1+N^{2}\right)}} .
$$

Preuve: Puisque $R(\lambda, \Gamma) \neq 0$, grâce à 2.6 on sait que si on choisit de façon convenable la base de $\mathcal{K}_{\lambda, \Gamma}^{(l)}$, la matrice $\mathcal{G}(\lambda, \Gamma)$ est de la forme

$$
\left(\begin{array}{ccccc}
G(\lambda, \Gamma) & & & & \\
& G(\lambda, \Gamma) & & \star & \\
& & \ddots & & \\
& O & & \ddots & \\
& & & & G(\lambda, \Gamma)
\end{array}\right)
$$

où la partie triangulaire supérieure est composée de matrice $N \times N$ à coefficients dans $K(\lambda, \Gamma)$ et où $G(\lambda, \Gamma)$ est une matrice $N \times N$ à coefficients dans $K(\lambda, \Gamma)$ qui représente 
la connexion $\sigma_{\Gamma}$ sur $\mathcal{W}_{\lambda, \Gamma}^{(1)}$. D'après A.1, si $R(\lambda, t) \neq 0$ alors $\frac{\partial}{\partial \Gamma}-G(\lambda, \Gamma)=0$ admet un système de solutions formelles dans $K(\lambda)[[\Gamma-t]]$, et donc les singularités non triviales de ce système sont contenues dans l'ensemble des $\Gamma$ tels que $R(\lambda, \Gamma)=0$. Mais alors si $\Gamma=\alpha \in \Omega$ est le zéro minimal de $R(\lambda, \Gamma)$ tel que $0<|\alpha| \leqslant 1$ (si un tel zéro n'existe pas on pose $\alpha=1$ ), on a que le système $\Gamma \frac{\partial}{\partial \Gamma}-\alpha \Gamma G(\lambda, \alpha \Gamma)=0$ a seulement des singularités triviales dans le disque $D\left(0,1^{-}\right) \backslash\{0\}$. D'autre part si on spécialise $\lambda$ dans un élément $\bar{\lambda}$ de $K$ tel que $R(\bar{\lambda}, \Gamma) \neq 0, \frac{\partial}{\partial \Gamma}-G(\bar{\lambda}, \Gamma)=0$ est "un" système (dont la variable est $\Gamma$ ), et donc grâce à $2.3 \rho(G(\bar{\lambda}, \Gamma))<+\infty$. Mais alors d'après [8, VII 2], pour une infinité de valeurs de $\lambda$ dans $K$, ce système a en $\Gamma=0$ une singularié régulière avec exposants rationnels, et par conséquent on peut conclure qu'aussi pour $\lambda$ transcendant sur $K, \frac{\partial}{\partial \Gamma}-G(\lambda, \Gamma)=0$ a en $\Gamma=$ 0 une singularité régulière avec exposants rationnels. Ceci implique que le système différentiel $\Gamma \frac{\partial}{\partial \Gamma}-\alpha \Gamma G(\lambda, \alpha \Gamma)=0$ a une matrice solution à $\Gamma=0$ de la forme suivante: $Y(\alpha \Gamma) \Gamma^{B}$ avec $Y(\Gamma) \in G L(N, K(\lambda)((\Gamma))), B \in M_{N \times N}(K(\lambda))$ et les valeurs propres de $B$ rationnels. Alors grâce à $\left[8\right.$, VI 3.4] $r(Y) \geqslant|\alpha| r\left(\mathcal{U}_{\alpha \Gamma G(\lambda, \alpha \Gamma), t_{v}}\right)^{N^{2}}$, où $\mathcal{U}_{\alpha \Gamma G(\lambda, \alpha \Gamma), t_{v}}$ est la matrice solution au point générique $t_{v}$ de $\Gamma \frac{\partial}{\partial \Gamma}-\alpha \Gamma G(\lambda, \alpha \Gamma)=0$ et $r$ indique le rayon de convergence. Par conséquent si $\bar{Y}(\Gamma) \Gamma^{\bar{B}}$, avec $\bar{Y}(\Gamma) \in G L(\bar{N}, K(\lambda)((\Gamma)))$ et $\bar{B} \in M_{\bar{N} \times \bar{N}}(K(\lambda))$, est une matrice solution à $\Gamma=0$ du système $\frac{\partial}{\partial \Gamma}-\mathcal{G}(\lambda, \Gamma)=0$, d'après $A .3$ on obtient

$$
r(\bar{Y}) \geqslant|\alpha| r\left(\mathcal{U}_{\alpha \Gamma G(\lambda, \alpha \Gamma), t_{v}}\right)^{N^{2}}
$$

On doit donc calculer $|\alpha|$ et $r\left(\mathcal{U}_{\alpha \Gamma G(\lambda, \alpha \Gamma), t_{v}}\right)$. Pour cela, on va reprendre partiellement la démonstration de [8, VII 3.3]: soit $t_{\alpha}$ un point générique tel que $\left|t_{\alpha}\right|=|\alpha|$. Si $t_{v}=\left(t_{\alpha} / \alpha\right)$ on trouve

$$
\mathcal{U}_{\alpha \Gamma G(\lambda, \alpha \Gamma), t_{v}}=\sum_{s=0}^{\infty} \alpha^{s} \frac{G_{s}\left(t_{\alpha}\right)}{s !}\left(\Gamma-t_{v}\right)^{s}
$$

Mais d'après [8, VII 3.1] on a

$$
\log \frac{\left.\frac{X^{s} G_{s}}{s !}\right|_{0}(1)}{\left|\frac{X^{s} G_{s}}{s !}\right|_{0}(|\alpha|)} \geqslant-s \sum_{\substack{0<|\Gamma|<1, \Gamma \text { non triviale } \\ R(\lambda, \Gamma)=0}} \log \frac{1}{\sup (|\alpha|,|\Gamma|)}+\text { constante }
$$


et donc grâce à (3.9.2), (3.9.3) et au théorème de Dwork-Robba [8, IV 2.1 et 2.2] on obtient

$$
r(\bar{Y}) \geqslant|\alpha| r\left(\mathcal{U}_{G(\lambda, \Gamma), t_{v}}\right)^{N^{2}}\left(\prod_{\substack{0<\{\Gamma \mid<1, \Gamma \text { non triviale } \\ R(\lambda, \Gamma)=0}}|\Gamma|\right)^{N^{2}}
$$

Par conséquent grâce à A.1 et A.2 on peut conclure que

$$
r(\bar{Y}) \geqslant\left|\rho_{0}\left(\lambda, A^{(0)}\right)\right|^{N^{2}+1}
$$

3.10. Avant d'utiliser les informations qu'on a trouvées sur $\vec{\theta}$, pour étudier $\mathcal{K}_{\lambda, 0}^{(l)}$, on va modifier les espaces $L(b)$ et $L^{*}(b)$, introduits dans 1.6 , en remplaçant $\Omega$ par $\Omega_{v}^{\prime}$, un corps qui soit complet pour une extension de la valeur absolue $v$-adique de $K\left(\pi_{v}\right)$ et qui contienne un point générique $t_{v}$. On considère $\lambda$ et $\Gamma$ comme des variables de $\Omega_{v}^{\prime}$. Nous allons chercher un élément $b \in \mathbb{R}, b>0$ tel que $\mathcal{K}_{\lambda, 0}^{(l)} \subset L^{*}(b)$. Cette information nous sera utile pour calculer $\rho(\mathcal{G}(\lambda))$.

THÉORĖme 3.11. $\mathcal{K}_{\lambda, 0}^{(l)} \subset L^{*}\left(\left(1+e\left(1+N^{2}\right)\right) \operatorname{ord} \rho_{0}\left(\lambda, A^{(0)}\right)\right)$.

Preuve: Puisque $R(\lambda, \Gamma) \neq 0$, d'après [5, Théorème 6.2] on sait que les éléments $w_{i, u, \lambda, \Gamma}^{*}$ de la base de $\mathcal{K}_{\lambda, \Gamma}^{(l)}$ peuvent s'écrire de la façon suivante:

$$
w_{i, u, \lambda, \Gamma}^{*}=\sum_{w \in \mathcal{F}_{0}} \frac{1}{\chi(\lambda, \Gamma)} \frac{G_{u, w, i}(\lambda, \Gamma)}{\left(R(\lambda, \Gamma) \pi_{v}\right)^{w_{0}}} \frac{1}{X^{w}}
$$

où $\chi(\lambda, \Gamma), R(\lambda, \Gamma)$ et $G_{u, w, i}(\lambda, \Gamma) \in \mathcal{O}_{K}[\lambda, \Gamma]$ pour $i=1, \ldots, N$ et pour tout $u$ tel que $0 \leqslant|u| \leqslant l-1$. Par conséquent si $\chi(\lambda, \Gamma)=\Gamma^{\tau} \cdot \bar{\chi}(\lambda, \Gamma)$ avec $\bar{\chi}(\lambda, 0) \neq 0$, d'après (3.6.1) et (3.6.2) on a

$$
\bar{\chi}(\lambda, \Gamma) w_{\lambda, 0}^{*}=\sum_{w \in \mathcal{F}_{0}} \frac{1}{X^{w}} C_{w}(\lambda)
$$

où

$$
C_{w}(\lambda)=
$$

$$
\left(\sum_{i=1}^{N} \sum_{0 \leqslant|u| \leqslant l-1} \frac{G_{u, w, i}(\lambda, \Gamma)}{\pi_{v}^{w_{0}}} \frac{\sum_{0 \leqslant s \leqslant e w_{0}+\tau} h_{i, u, s} \Gamma^{s}}{\Gamma^{e w_{0}+\tau}\left(\rho_{0}\left(\lambda, A^{(0)}\right)+\Gamma \rho_{1}\left(\lambda, A^{(0)}\right)+\ldots\right)^{w_{0}}}\right)_{\mid \Gamma=0} .
$$

Donc, pour trouver un réel $b$ tel que $\mathcal{K}_{\lambda, 0}^{(l)} \subset L^{*}(b)$, il suffit de calculer une estimation de $\operatorname{ord} C_{u}(\lambda)$. En utilisant les notations de 3.9 , on observe que

$$
\left(\rho_{0}\left(\lambda, A^{(0)}\right)+\Gamma \rho_{1}\left(\lambda, A^{(0)}\right)+\ldots\right)^{-w_{0}}
$$


est analytique pour $|\Gamma|<|\alpha|$ et limité dans $D\left(0,|\alpha|^{-}\right)$par $1 /\left(\left|\rho_{0}\left(\lambda, A^{(0)}\right)\right|^{w_{0}}\right)$. Alors d'après Cauchy on a

$$
\left(\rho_{0}\left(\lambda, A^{(0)}\right)+\Gamma \rho_{1}\left(\lambda, A^{(0)}\right)+\ldots\right)^{-w_{0}}=q_{0}\left(w_{0}\right)+q_{1}\left(w_{0}\right) \Gamma+\ldots
$$

avec $\left|q_{j}\left(w_{0}\right)\right| \leqslant 1 /\left(\left|\rho_{0}\left(\lambda, A^{(0)}\right)\right|^{w_{0}}|\alpha|^{j}\right)$ et donc grâce à A.2 on obtient

$$
\left|q_{j}\left(w_{0}\right)\right| \leqslant \frac{1}{\left|\rho_{0}\left(\lambda, A^{(0)}\right)\right|^{w_{0}+j}} .
$$

Par conséquent en spécialisant de façon convenable le vecteur $A$ de 1.8 et en utilisant (3.11.2) et (3.9.1), d'après (3.11.1) on peut enfin conclure que

$$
\left|C_{w}(\lambda)\right| \leqslant p^{\tilde{\varepsilon} w_{0}} \frac{M(\varepsilon)}{\varepsilon^{\left(\tau+e w_{0}\right)\left(1+N^{2}\right)}} \frac{1}{\left|\rho_{0}\left(\lambda, A^{(0)}\right)\right|^{\left(1+e\left(1+N^{2}\right)\right) w_{0}+\tau\left(1+N^{2}\right)}} .
$$

3.12. Maintenant qu'on a des informations précises sur $\mathcal{K}_{\lambda, 0}^{(l)}$, on peut finalement chercher le rayon de convergence de la matrice solution de $\frac{\partial}{\partial \lambda}-\mathcal{G}(\lambda)=0$ au point générique $t_{v}$ et donc conclure enfin que, même dans le cas d'une suite non régulière, $\mathcal{W}^{(l)}$ est de type $G$ pour chaque $l \geqslant 1$.

ThÉoRÈme 3.13. Soit $f_{1}, \ldots, f_{n+1}$ une suite non régulière. Alors, pour chaque $v \in \mathcal{P}_{f}$ le rayon de convergence, $R_{v}$, de la matrice solution de $\frac{\partial}{\partial \lambda}-G(\lambda)=0$ au point générique $t_{v}$, est au moins $\left|\rho_{0}\left(t_{v}, A^{(0)}\right)\right|^{\left(1+e\left(N^{2}+1\right)\right)}$. Par conséquent

$$
\rho(\mathcal{G}(\lambda)) \leqslant\left(1+e\left(N^{2}+1\right)\right) \sum_{v \in \mathcal{P}_{f}} \log \frac{1}{\left|\rho_{0}\left(\lambda, A^{(0)}\right)\right|_{v}} .
$$

PREUVE: La démonstration est analogue à celle de 2.3. En effet, si on choisit un point générique $t_{v}$ tel que $\rho_{0}\left(t_{v}, A^{(0)}\right) \neq 0$, pour trouver la matrice solution de $\frac{\partial}{\partial \lambda}-\mathcal{G}(\lambda)=0$ en ce point, on utilise l'application $T_{t_{v}, \lambda}^{*}$ définie en 2.3 , mais ici on va la faire agir entre les espaces $\mathcal{K}_{t_{v}, 0}^{(l)}$ et $\mathcal{K}_{\lambda, 0}^{(l)} \otimes \Omega_{v}$, c'est-à-dire,

$$
T_{t_{v}, \lambda}^{*}: \mathcal{K}_{t_{v}, 0}^{(l)} \longrightarrow \mathcal{K}_{\lambda, 0}^{(l)} \otimes \Omega_{v}
$$

Après les calculs sont similaires à ceux de 2.3: il suffit de remplacer (2.3.2) par 3.11. 


\section{Majoration DE $\rho(\mathcal{G})$}

4.0. Dans ce paragraphe on va améliorer (3.13.1) en trouvant une bonne majoration pour $\sum_{v \in \mathcal{P}_{f}} \log 1 /\left(\left|\rho_{0}\left(\lambda, A^{(0)}\right)\right|_{v}\right)$ en fonction de $n, d, m$ et $h_{\infty}(f)$.

4.1. Commençons par spécialiser $\lambda, \Gamma$ et par modifier la spécialisation $\dot{A}^{(0)}$ de $A$ faite en 3.1.

En prolongeant éventuellement le corps $K$ si nécessaire, spécialisons $\lambda$ en une racine de l'unité, soit $\xi$, telle que $\rho_{0}\left(\xi, A^{(0)}\right) \neq 0$. Puisque $g(\lambda, \Gamma, X) \in \mathcal{O}_{K}[\lambda, \Gamma, X]$ et $\xi$ est une racine de l'unité, on a

$$
\sum_{v \in \mathcal{P}_{f}} \log \frac{1}{\left|\rho_{0}\left(\xi, A^{(0)}\right)\right|_{v}}=\sum_{v \in \mathcal{P}_{\infty}} \log \left|\rho_{0}\left(\xi, A^{(0)}\right)\right|_{v}
$$

où la dernière égalité est due à la formule du produit [8, VII 1]. Spécialisons maintenant $\Gamma$ : soit $\mu \in K$ tel que $|\mu|_{v}=1$ pour tout $v \in \mathcal{P}_{\infty}$ et $R(\xi, \mu) \neq 0$. En ce qui concerne $A^{(0)}$ on va modifier cette spécialisation de $A$ de façon à ce que:

(1) $A^{(0)} \in \mathcal{O}_{K}^{\left(\begin{array}{c}n+d \\ n\end{array}\right)}$

(2) $\rho_{0}\left(\lambda, A^{(0)}\right) \neq 0$,

(3) les coefficients $A_{u}^{(0)}$ soient des racines de l'unité.

Pour simplifier les notations posons

$$
f(\lambda, X)=\sum_{u \in \mathcal{F}} \sum_{s=0}^{m} C_{u, s} \lambda^{s} X^{u} \quad, \quad h\left(A^{(0)}, X\right)=\sum_{u \in \mathcal{F}} A_{u}^{(0)} X^{u},
$$

et $H_{v}=\sup _{\substack{u \in \mathcal{F} \\ 0 \leqslant \leqslant \leqslant m}}\left|C_{u, s}\right|_{v}$ pour tout $v \in \mathcal{P}$. Avec ces spécialisations de $\lambda, \Gamma$ et $A$, on va maintenant améliorer (4.1.1) et donc (3.13.1).

THÉORÈme 4.2 .

$$
\begin{gathered}
\rho(\mathcal{G}(\lambda)) \leqslant d^{2 n}\left(\begin{array}{c}
n+(n+1) d \\
n
\end{array}\right)^{2} . \\
{\left[\frac{3}{2} \log \left(\left(\begin{array}{l}
n+d \\
d-2
\end{array}\right) \frac{4(n+2 d)}{(d-1)}\right)+\frac{3}{4}\left(\log (m+1)+h_{\infty}(f)\right)\right] .}
\end{gathered}
$$

Preuve: Puisque $R(\xi, \mu) \neq 0$, d'après [5, Section 1] l'application linéaire

$$
\begin{gathered}
\delta: R_{n}^{n+1} \longrightarrow R_{n+1} \\
\left(q_{1}, \ldots, q_{n+1}\right) \longmapsto \sum_{i=1}^{n+1} q_{i} g_{i}
\end{gathered}
$$


est surjective. Choisissons comme bases de $R_{n+1}$ et de $R_{n}^{n+1}$ celles composées de monômes. Conformément à $3.1 \rho_{0}\left(\xi, A^{(0)}\right)$ est le coefficient de $\mu^{e}$ dans $R(\xi, \mu)$, et $R(\xi, \mu)$ est le déterminant de la sous matrice de rang maximum de la matrice $\Delta$ qui représente $\delta$ par rapport aux bases qu'on a choisies. Par conséquent en utilisant les notations de 4.1 on trouve

$$
\rho_{0}\left(\xi, A^{(0)}\right)=\sum_{\substack{J \subseteq I, \# J=e \\ J \cup J^{\prime}=I, J \cap J^{\prime}=0}}\left(\wedge_{\alpha \in J^{\prime}} \overrightarrow{\mathcal{X}}_{\alpha}\right) \wedge\left(\wedge_{\alpha \in J} \overrightarrow{\mathcal{Y}}_{\alpha}\right)
$$

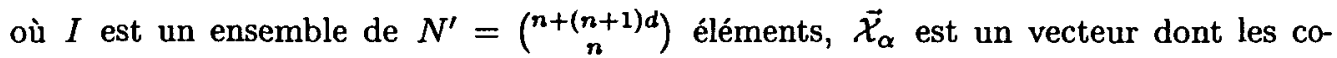
efficients sont les $u_{i}\left(\sum_{s=0}^{m} C_{u, s} \xi^{s}\right)$ pour tout $u \in \mathcal{F}$ et $\overrightarrow{\mathcal{Y}}_{\alpha}$ est un vecteur dont les coefficients sont les $u_{i} A_{u}^{(0)}$ pour tout $u \in \mathcal{F}$. Comme d'autre part

$$
\sum_{u \in \mathcal{F}} u_{i}^{2}=\left(\begin{array}{l}
n+d \\
d-1
\end{array}\right)+2\left(\begin{array}{l}
n+d \\
d-2
\end{array}\right)=\left(\begin{array}{l}
n+d \\
d-2
\end{array}\right) \frac{(n+2 d)}{(d-1)}
$$

et que $\xi$ et les coefficients du vecteur $A^{(0)}$ sont des racines de l'unité, on trouve

$$
\begin{aligned}
& \left\|\overrightarrow{\mathcal{Y}}_{\alpha}\right\| \leqslant\left(\left(\begin{array}{l}
n+d \\
d-2
\end{array}\right) \frac{(n+2 d)}{(d-1)}\right)^{1 / 2}, \\
& \left\|\overrightarrow{\mathcal{X}}_{\alpha}\right\| \leqslant\left(\left(\begin{array}{l}
n+d \\
d-2
\end{array}\right) \frac{(n+2 d)}{(d-1)}\right)^{1 / 2}(m+1) H_{v, \mathrm{cl}},
\end{aligned}
$$

où pour tout $v \in \mathcal{P}_{\infty}, H_{v, \mathrm{cl}}=H_{v}^{l / \varepsilon_{v}}$ avec $l=[K: \mathbb{Q}], \varepsilon_{v}=1$ si $K_{v}=\mathbb{R}$ et $\varepsilon_{v}=2$ si $K_{v}=\mathbb{C}$. Mais alors, en appliquant le théorème d'Hadamard aux $\left(\begin{array}{c}N^{\prime} \\ e\end{array}\right)$ déterminants qui apparaissent dans la formule (4.2.2), on obtient grâce à (4.2.3), (4.2.4), (4.1.1) et (3.13.1) que

$$
\begin{aligned}
\rho(\mathcal{G}(\lambda)) \leqslant & \left(1+e\left(N^{2}+1\right)\right)\left[\log \left(\begin{array}{c}
N^{\prime} \\
e
\end{array}\right)\right. \\
& \left.+\frac{N^{\prime}}{2} \log \left(\left(\begin{array}{l}
n+d \\
d-2
\end{array}\right) \frac{(n+2 d)}{(d-1)}\right)+\left(N^{\prime}-e\right)\left(\log (m+1)+h_{\infty}(f)\right)\right] .
\end{aligned}
$$

Enfin puisque $\left(\begin{array}{c}N^{\prime} \\ e\end{array}\right) \leqslant 2^{N^{\prime}}, e\left(N^{\prime}-e\right) \leqslant\left(N^{\prime} / 2\right)^{2}$ et $e \leqslant N^{\prime}$, on trouve la majoration (4.2.1).

REMARQUE 4.3. On observe que la formule (4.2.1) nous fournit une majoration effective de $\rho(\mathcal{G}(\lambda))$ indépendante de "l ". En effet la majoration, que nous venons de trouver, dépend seulement du polynôme $f(\lambda, X)$ introduit dans 0.3 :

(1) $n+1$ est le nombre de variables $X_{1}, \ldots, X_{n+1}$ de $f(\lambda, X)$, 
(2) $d$ est le degré d'homogénéité en les variables $X_{1}, \ldots, X_{n+1}$ de $f(\lambda, X)$,

(3) $m$ est le degré en $\lambda$ de $f(\lambda, X)$,

(4) $h_{\infty}(f)$ est la hauteur logarithmique à l'infini de $f(\lambda, X)$.

De plus grâce à (4.2.5) on a que si $f_{1}, \ldots, f_{n+1}$ est une suite régulière $(e=0)$, alors

$$
\rho(\mathcal{G}(\lambda)) \leqslant N^{\prime}\left(\frac{1}{2} \log \left(\left(\begin{array}{l}
n+d \\
d-2
\end{array}\right) \frac{(n+2 d)}{(d-1)}\right)+\log (m+1)+h_{\infty}(f)\right) \stackrel{d e ́ f}{=} \rho_{0}(\mathcal{G}(\lambda))
$$

et si $f_{1}, \ldots, f_{n+1}$ n'est pas une suite régulière $(e \neq 0)$, alors

$$
\rho(\mathcal{G}(\lambda)) \leqslant\left(1+e\left(N^{2}+1\right)\right)\left[\log \left(\begin{array}{c}
N^{\prime} \\
e
\end{array}\right)+\rho_{0}(\mathcal{G}(\lambda))\right] \stackrel{\text { déf }}{=} \rho_{e}(\mathcal{G}(\lambda)) .
$$

\section{EXEMPLE}

5.1. Je termine en donnant un exemple de module $\mathcal{W}^{(l)}$. Soit

$$
f\left(\lambda, X_{1}, X_{2}\right)=X_{1}^{2}+2 \lambda X_{1} X_{2}+X_{2}^{2}
$$

En utilisant la terminologie de la Section 0 on trouve que

$$
\begin{aligned}
& D_{1}=E_{1}+2\left(X_{1}^{2}+\lambda X_{1} X_{2}\right), \\
& D_{2}=E_{2}+2\left(\lambda X_{1} X_{2}+X_{2}^{2}\right), \\
& \sigma_{\lambda}=\frac{\partial}{\partial \lambda}+2 X_{1} X_{2} .
\end{aligned}
$$

Puisque d'après $0.3 R=\bigoplus_{s \in \mathbb{N}} R_{s}$, où $R_{s}$ est l'espace engendré sur $K(\lambda)$ par les monômes $X_{1}^{u_{1}} X_{2}^{u_{2}}$ avec $u_{1}+u_{2}=s 2$, on a que $R$ est le $K(\lambda)$-espace des polynômes homogènes de degré pair dans les variables $X_{1}$ et $X_{2}$. En particulier $R=\left(f_{1} R+f_{2} R\right)+1 K(\lambda)+$ $X_{1} X_{2} K(\lambda)$ et donc $\mathcal{W}^{(1)}=R /\left(D_{1} R+D_{2} R\right)$ est engendré sur $K(\lambda)$ par $1 \bmod D_{1} R+$ $D_{2} R$ et $X_{1} X_{2} \bmod D_{1} R+D_{2} R$.

Maintenant cherchons la matrice $G(\lambda)$ qui représente la connexion $\sigma_{\lambda}$ sur $\mathcal{W}^{(1)}$, c'est-à-dire, telle que

$$
\sigma_{\lambda}\left(\begin{array}{c}
1 \\
X_{1} X_{2}
\end{array}\right)=G(\lambda)\left(\begin{array}{c}
1 \\
X_{1} X_{2}
\end{array}\right) \quad \bmod D_{1} R+D_{2} R
$$

On observe que

$$
\sigma_{\lambda}\left(\begin{array}{c}
1 \\
X_{1} X_{2}
\end{array}\right)=\left(\begin{array}{c}
2 X_{1} X_{2} \\
2 X_{1}^{2} X_{2}^{2}
\end{array}\right)
$$


En utilisant les définitions de $f_{1}$ et de $f_{2}$ et la règle de Cramer on trouve

$$
2 X_{1}^{2} X_{2}^{2}=\frac{2}{1-\lambda^{2}}\left(X_{1}^{2} \frac{1}{2} f_{2}-X_{1} X_{2} \frac{1}{2} f_{1} \lambda\right) \text {. }
$$

Mais puisque

$$
\begin{aligned}
X_{1}^{2} f_{2} & \equiv 0 & & \bmod D_{1} R+D_{2} R \\
X_{1} X_{2} f_{1} & \equiv-X_{1} X_{2} & & \bmod D_{1} R+D_{2} R
\end{aligned}
$$

on obtient

$$
G(\lambda)=\left(\begin{array}{cc}
0 & 2 \\
0 & \frac{\lambda}{1-\lambda^{2}}
\end{array}\right) .
$$

5.2. Pour avoir des informations sur $\mathcal{W}^{(l)}$ pour $l>1$, on observe que

$$
R(\lambda)=16\left(1-\lambda^{2}\right) \neq 0,
$$

c'est-à-dire, $f_{1}, f_{2}$ est une suite régulière. Par conséquent, pour étudier $\mathcal{W}^{(l)}$ on peut utiliser les résultats obtenus dans la Section 2. En particulier grâce à 2.5 on a

$$
\operatorname{dim}_{K(\lambda)} \mathcal{W}^{(l)}=l(l+1)
$$

et d'après 2.6 on sait que si on choisit de façon convenable la base de $\mathcal{W}^{(l)}$ alors la matrice $\mathcal{G}(\lambda)$, qui représente la connexion $\sigma_{\lambda}$ sur $\mathcal{W}^{(l)}$, est de la forme

$$
\left(\begin{array}{ccccccc}
0 & 2 & & & & & \\
0 & \frac{\lambda}{1-\lambda^{2}} & & & & \star & \\
& & 0 & 2 & & & \\
& & 0 & \frac{\lambda}{1-\lambda^{2}} & & & \\
& & & & \ddots & & \\
& 0 & & & & 0 & 2 \\
& & & & & 0 & \frac{\lambda}{1-\lambda^{2}}
\end{array}\right)
$$

où la "diagonale" est composée de $l(l-1) / 2$ matrices $2 \times 2$ toutes égales à $G(\lambda)$. De plus puisque $h_{\infty}(f)=\log 2$, d'après (4.3.1) on a

$$
\rho_{0}(\mathcal{G}(\lambda)) \leqslant 5\left(\frac{1}{2} \log 5+2 \log 2\right)<+\infty .
$$

Mais dans cet exemple très simple, on peut vraiment calculer $\rho(\mathcal{G}(\lambda))$. En effet, les solutions du système $d /(d \lambda)-G(\lambda)=0$ sont

$$
\left(\begin{array}{l}
1 \\
0
\end{array}\right), \quad\left(\begin{array}{c}
\int \frac{2}{\left(1-\lambda^{2}\right)^{1 / 2}} d \lambda \\
\frac{1}{\left(1-\lambda^{2}\right)^{1 / 2}}
\end{array}\right)
$$

et puisque l'intégration ne change pas le rayon de convergence, celui-ci est 1 si $p \neq 2$ et $1 / 2$ pour $p=2$. Par conséquent $\rho(\mathcal{G}(\lambda))=2$. 


\section{APPENDICE}

Proposition A.1. En utilisant les notations de la Section 3 , soient $v \in \mathcal{P}_{f}, t_{v}$ un point générique pour cette valeur absolue et $R\left(\lambda, t_{v}\right)$ le résultant de $g_{1}\left(\lambda, t_{v}, X\right), \ldots$, $g_{n+1}\left(\lambda, t_{v}, X\right)$. De plus soit $G(\lambda, \Gamma)$ la matrice $N \times N$ à coefficients dans $K(\lambda, \Gamma)$ qui représente la connexion $\sigma_{\Gamma}=\frac{\partial}{\partial \Gamma}+X_{0} \pi \frac{\partial g}{\partial \Gamma}$ sur $\mathcal{W}_{\lambda, \Gamma}^{(1)}$. Alors si $R\left(\lambda, t_{v}\right) \neq 0$, les solutions du système $\frac{\partial}{\partial \Gamma}-G(\lambda, \Gamma)=0$ au point générique $t_{v}$, convergent pour $\left|\Gamma-t_{v}\right|<\left|R\left(\lambda, t_{v}\right)\right|$.

Preuve: Analogue à celle de 2.3 Section 2.

LEMmE A.2. Soit $\Omega$ un corps algébriquement clos muni d'une valeur absolue finie. Soit $f(X)=A_{0}+A_{1} X+\cdots+A_{m} X^{m} \in \Omega[X]$ avec $f(0)=A_{0} \neq 0$. Alors

$$
\prod_{\substack{f(\gamma)=0 \\|\gamma|<1}}|\gamma|=\frac{\left|A_{0}\right|}{|f|_{\text {gauss }}} .
$$

Preuve: Conséquence de [8, I 6.1].

Lemme A.3. Soient $F$ un corps différentiel de caractéristique zéro muni d'une valeur absolue qui prolonge la valeur absolue $p$-adique de $\mathbb{Q}, X$ un élément transcendant sur $F$ et $\delta=X \frac{\mathrm{d}}{\mathrm{d} X}$. Posons $S=F((X))$ et

$$
S_{1}=\{\xi \in S \mid \xi \text { analytique pour } 0<|X|<1\} .
$$

De plus soit $G$ une matrice appartenant à $M_{r n \times r n}\left(S_{1}\right)$ de la forme suivante

$$
G=\left(\begin{array}{ccccc}
G_{1} & & & & \\
& G_{1} & & \star & \\
& & \ddots & & \\
& o & & G_{1} & \\
& & & & G_{1}
\end{array}\right)
$$

avec $G_{1} \in M_{n \times n}\left(S_{1}\right)$ tel que:

(1) $\delta-G_{1}$ a comme matrice solution $U_{1} X^{A_{1}}$ avec $U_{1} \in G L\left(n, S_{1}\right)$ et $A_{1} \in$ $M_{n \times n}(F)$,

(2) la différence entre les valeurs propres de $A_{1}$ est de type 1 (voir [8, VI 1] pour la définition de "nombre de type 1").

Alors le système $\delta-G$ a une matrice solution de la forme $U X^{B}$ avec $U \in G L\left(r n, S_{1}\right)$ et $B \in M_{r n \times r n}(F)$.

PREUVE: Je vais seulement donner l'ésquisse de cette démonstration. 
I. Supposons que $\mathcal{F}$ contienne

(1) $q$ éléments $z_{1}, \ldots, z_{q}$ tels que $\forall i, j=1, \ldots q, \delta\left(z_{i}\right)=\alpha_{i} z_{i}$ avec $\alpha_{i} \in F$ et $\alpha_{i}-\alpha_{j} \notin \mathbb{Z}$ pour $i \neq j$,

(2) un élément $w$ tel que $\delta(w)=1$.

Alors les $\left\{z_{i} w^{j}\right\}_{\substack{1 \leqslant i \leqslant q \\ j \geqslant 0}}$ sont linéairement indépendant sur $F((X))$.

II. Posons $T=S[\log X], T_{1}=S_{1}[\log X]$ et $\forall \alpha \in F \quad W_{\alpha}=X^{\alpha} T$ et $W_{\alpha, 1}=$ $X^{\alpha} T_{1}$. De plus soient $\alpha_{1}, \ldots, \alpha_{q}$ des éléments de $\mathrm{F}$ appartenant à des classes distinctes modulo $\mathbb{Z}$. Alors la somme $W_{\alpha_{1}}+\cdots+W_{\alpha_{q}}$ est directe et si $-\alpha_{1}, \ldots,-\alpha_{q}$ sont de type 1 , alors

$$
\delta\left(W_{\alpha_{1}, 1}+\cdots+W_{\alpha_{q}, 1}\right)=W_{\alpha_{1}, 1}+\cdots+W_{\alpha_{q}, 1}
$$

III. Soit $\bar{G}$ une matrice appartenant à $M_{\left(n_{1}+n_{4}\right) \times\left(n_{1}+n_{4}\right)}(F((X)))$ de la forme suivante:

$$
\bar{G}=\left(\begin{array}{cc}
\bar{G}_{1} & \bar{G}_{2} \\
O & \bar{G}_{4}
\end{array}\right)
$$

avec $\bar{G}_{1} \in M_{n_{1} \times n_{1}}(F[[X]])$ et $\bar{G}_{4} \in M_{n_{4} \times n_{4}}(F[[X]])$. Alors il existe une matrice $H \in G L\left(n_{1}+n_{4}, F(X)\right)$ tel que

$$
\bar{G}_{[H]}=X \frac{\partial}{\partial X}(H) H^{-1}+H \bar{G} H^{-1} \in M_{\left(n_{1}+n_{4}\right) \times\left(n_{1}+n_{4}\right)}(F[[X]])
$$

IV. Soit $\widetilde{G}$ une matrice appartenant à $M_{\left(n_{1}+N_{4}\right) \times\left(n_{1}+N_{4}\right)}\left(S_{1}\right)$ de la forma suivante:

$$
\tilde{G}=\left(\begin{array}{cc}
\widetilde{G}_{1} & \widetilde{G}_{2} \\
O & \widetilde{G}_{4}
\end{array}\right)
$$

Supposons que $\delta-\widetilde{G}_{1}$ (respectivement $\delta-\widetilde{G}_{4}$ ) ait comme matrice solution $\mathcal{U}_{1} X^{\tilde{A}_{1}}$ (respectivement $\mathcal{U}_{4} X^{\widetilde{A}_{4}}$ ), avec $\mathcal{U}_{1} \in G L\left(n_{1}, S_{1}\right)$ et $\widetilde{A}_{1} \in M_{n_{1} \times n_{1}}(F)$ (respectivement $\mathcal{U}_{4} \in G L\left(n_{4}, S_{1}\right)$ et $\left.\tilde{A}_{4} \in M_{n_{4} \times n_{4}}(F)\right)$, et que les differences $\alpha-\beta$, avec $\alpha$ et $\beta$ respectivement valeurs propres de $\widetilde{A}_{1}$ et $\widetilde{A}_{4}$, soient de type 1 . Alors $\delta-\widetilde{G}$ admet comme matrice solution $\mathcal{U} X^{\widetilde{B}}$, avec $\mathcal{U} \in G L\left(n_{1}+n_{4}, S_{1}\right)$ et $\widetilde{B} \in M_{\left(n_{1}+N_{4}\right) \times\left(n_{1}+N_{4}\right)}(F)$.

Index des notations

$\begin{array}{llll}m & 0.3 . & \Omega & 1.6 . \\ R & 0.3 . & R_{\lambda}^{*} & 2.2 . \\ d & 0.3 . & D_{\lambda, i}^{*} & 2.2 . \\ f(\lambda, X) & 0.3 . & \mathcal{K}_{\lambda}^{(1)} & 2.2 . \\ E_{i} & 0.3 . & \Omega_{v} & 2.2 . \\ f_{i} & 0.3 . & G(\lambda) & 2.2 .\end{array}$




\begin{tabular}{|c|c|c|c|}
\hline$D_{i}$ & 0.3 & $\Gamma$ & 3.0. \\
\hline$D^{v}$ & 0.3 & $g(\lambda, \Gamma, A, X)$ & 3.1. \\
\hline$R_{s}$ & 0.3 & $R(\lambda, \Gamma, A)$ & 3.1. \\
\hline $\mathcal{F}$ & 0.3 & $\rho_{i}(\lambda, A)$ & 3.1. \\
\hline $\mathcal{W}^{(l)}$ & 0.3 & $e$ & 3.1. \\
\hline$\sigma_{\lambda}$ & 0.3 & $A^{(0)}$ & 3.1. \\
\hline$h(A, X)$ & 1.0 & $g(\lambda, \Gamma, A)$ & 3.1 . \\
\hline$A$ & 1.1. & $R(\lambda, \Gamma)$ & 3.1. \\
\hline$K_{0}$ & 1.1. & $R_{\lambda, \Gamma}$ & 3.3. \\
\hline$\Omega_{0}$ & 1.1. & $R_{\lambda, \Gamma}^{*}$ & 3.3. \\
\hline$h_{i}$ & 1.1. & $D_{\lambda, \Gamma, i}$ & 3.3. \\
\hline$R\left(h, \ldots, h_{n}\right)$ & 1.2 & $D_{\lambda, \Gamma, i}^{*}$ & 3.3 \\
\hline$R_{A}$ & 1.4. & $\mathcal{W}_{\lambda, \Gamma}^{(l)}$ & 3.3. \\
\hline $\mathcal{W}_{A}^{(l)}$ & 1.4. & $\mathcal{K}_{\lambda, \Gamma}^{(l)}$ &. \\
\hline$D_{A, i}$ & $(1.4 .1)$ & $\mathcal{G}(\lambda)$ & 3.6. \\
\hline$D_{A}^{v}$ & 1.4. & $w_{\lambda, 0}^{*}$ & 3.6. \\
\hline$N$ & $(1.4 .2)$ & $\mathcal{K}_{\lambda, 0}^{(l)}$ & 3.6. \\
\hline $\bar{N}$ & $(1.4 .3)$ & $T_{0, \Gamma}^{*}$ & 3.6. \\
\hline$R_{A}^{*}$ & 1.5. & $w_{i, \lambda, \Gamma}$ & 3.6. \\
\hline$D_{A, i}^{*}$ & $(1.5 .1)$ & $\vec{\theta}=\left(\theta_{i, v}(\lambda, \Gamma)\right)$ & 3.6. \\
\hline$\gamma_{-}$ & 1.5 . & $w_{i, v, \lambda, \Gamma}^{*}$ & 3.6 \\
\hline $\mathcal{K}_{A}^{(i)}$ & 1.5 . & $\mathcal{G}(\lambda, \Gamma)$ & 3.0 \\
\hline $\mathcal{F}_{0}$ & 1.6. & $\sigma_{\Gamma}$ & 3.6. \\
\hline$L(b)$ & 1.6. & $\Omega_{v}^{\prime}$ & 3.10 . \\
\hline$L^{*}(b)$ & 1.6. & $\xi$ & 4.1 \\
\hline$L_{c}(b)$ & 1.6. & $\mu$ & 4.1 \\
\hline$L(b, c)$ & 1.6. & $H_{v}$ & 4.1 \\
\hline$M(b)$ & 1.6. & & \\
\hline
\end{tabular}

\section{BIBLIOGRAPHIE}

[1] Y. André, G-Functions and geometry, Aspects of Mathematics E13 (Friedr. Vieweg and Sohn, Braunschweig, 1989).

[2] André et Baldassarri, 'Geometric theory of G-functions', in Proceeding of Cortona's conference, (Catanese, Editor).

[3] B. Dwork, 'On the Zeta function of a hypersurface', Publications Mathématiques I.H.E.S 12 (1962), 5-68.

[4] B. Dwork, 'Zeta function of a hypersurface II', Ann. Math. 80 (1964), 227-299.

[5] B. Dwork, 'Zeta function of a hypersurface III', Ann. Math. 83 (1966), 457-519. 
[6] B. Dwork, Lectures on p-adic differential equations (Springer-Verlag, Berlin, Heidelberg, New York, 1982).

[7] B. Dwork, Generalized hypergeometric functions, Oxford Science Publications (Oxford University Press, New York, 1990).

[8] B. Dwork, G. Gerotto et F.J. Sullivan, An introduction to $G$-functions, Annals of Mathematics Studies 133 (Princeton University Press, 1994).

[9] B. Dwork et F. Loeser, 'Hypergeometric series', Japan. J. Math. 19 (1993), 81-129.

[10] N.M. Katz, 'On the differential equations satisfied by period matrices', Publications Mathématiques I.H.E.S 35 (1968), 223-258.

[11] J.P. Serre, 'Endomorphismes complètement continus des espaces de Banach p-adiques', Publications Mathématiques I.H.E.S 12 (1962), 69-85.

Institut de mathématiques, case 247

Université Pierre et Marie Curie

4 place Jussieu - F-75252

Paris, Cedex 05

France

e-mail: bertolin@riemann.math.jussieu.fr 